\title{
A Multifactor, Nonlinear, Continuous-Time Model of INTEREst Rate Volatility
}

\author{
Jacob Boudoukh $^{a}$, Matthew Richardson ${ }^{b}$, Richard Stanton $^{c}$ \\ and Robert F. Whitelaw ${ }^{d *}$ \\ June 17, 1999
}

\footnotetext{
${ }^{* a}$ Stern School of Business, NYU, 40 West 4th Street, Suite 9-160, New York, NY 10012, email: jboudouk@stern.nyu.edu; ${ }^{b}$ Stern School of Business, NYU and NBER, 40 West 4th Street, Suite 9-190, New York, NY 10012, email: mrichardson@stern.nyu.edu, phone \#: (212) 998-0349, fax \#: (212) 995-0888; ${ }^{c}$ Haas School of Business, U.C. Berkeley, 545 Student Services Building \#1900, Berkeley, CA 94720-1900, phone \#: (510) 642-7382, fax \# (510) 643-1420, email: stanton@haas.berkeley.edu; ${ }^{d}$ Stern School of Business, NYU, 40 West 4th Street, Suite 9-190, New York, NY 10012, email: rwhitela@stern.nyu.edu. We would like to thank John Cochrane, Lars Hansen, Chester Spatt, and seminar participants at the New York Federal Reserve, the Federal Reserve Board, Goldman Sachs, Long Term Capital Management, University of North Carolina, U.C. Berkeley, ITAM, the 1997 meetings of the Western Finance Association, the 1998 Utah Winter Finance Conference, and the NBER asset pricing program for helpful comments.
} 


\title{
A Multifactor, Nonlinear, Continuous-Time Model of INTEREST RATE Volatility
}

\begin{abstract}
This paper presents a general, nonlinear version of existing multifactor models, such as Longstaff and Schwartz (1992). The novel aspect of our approach is that rather than choosing the model parameterization out of "thin air", our processes are generated from the data using approximation methods for multifactor continuous-time Markov processes. In applying this technique to the short- and long-end of the term structure for a general two-factor diffusion process for interest rates, a major finding is that the volatility of interest rates is increasing in the level of interest rates only for sharply upward sloping term structures. In fact, the slope of the term structure plays a larger role in determining the magnitude of the diffusion coefficient. As an application, we analyze the model's implications for the term structure of term premiums.
\end{abstract}




\section{Introduction}

It is now widely believed that interest rates are affected by multiple factors. Part of this view derives from the fact that the returns on bonds of all maturities are not perfectly correlated. ${ }^{1}$ In addition to this simple point, a number of theoretical studies promote multifactor bond pricing, including Brennan and Schwartz (1979), Schaefer and Schwartz (1984), Heath, Jarrow and Morton (1988), Longstaff and Schwartz (1992), and Chen and Scott (1995), among others. Empirical studies of these and related models generally support the existence of multiple factors (see, for example, Dai and Singleton (1997), Litterman and Scheinkman (1991), Longstaff and Schwartz (1992), Stambaugh (1988), Pearson and Sun (1989), and Andersen and Lund (1997)). Despite this volume of evidence, surprisingly few stylized facts are known about the stochastic behavior of interest rates in a multi-factor, continuous-time setting.

This lack of evidence is particularly unfortunate as most of our intuition concerning bond and fixed-income derivative pricing comes from stylized facts generated by singlefactor, continuous-time interest rate models. For example, the finance literature is uniform in its view that interest rate volatility is increasing in interest rate levels, though there is some disagreement about the rate of increase (see, for example, Chan, Karolyi, Longstaff and Sanders (1992), Ait-Sahalia (1996b), Conley, Hansen, Luttmer and Scheinkman (1995), Brenner, Harjes and Kroner (1996) and Stanton (1997)). If interest rates possess multiple factors, such as the level and slope of the term structure (Litterman and Scheinkman (1991)), then this volatility result represents an average over all possible term structure slopes. Therefore, conditional on any particular slope, volatility may be severely misestimated, with serious consequences especially for fixed-income derivative pricing.

Two issues arise in trying to generate stylized facts about the underlying continuous-time, stochastic process for interest rates. First, how do we specify ex ante the drift and diffusion of the multivariate process for interest rates so that it is consistent with the true process underlying the data? Second, given that we do not have access to continuous-time data, but instead to interest rates/bond prices at discretely sample intervals, how can we consistently infer an underlying continuous-time multivariate process from these data? Recently, in singlefactor settings, there has been much headway at addressing these issues (see, for example, Ait-Sahalia (1996a), Conley, Hansen, Luttmer and Scheinkman (1995) and Stanton (1997)).

\footnotetext{
${ }^{1}$ In a single factor world, the instantaneous returns on all interest rate dependent assets must be perfectly correlated.
} 
Essentially, using variations on nonparametric estimators with carefully chosen moments, the underlying single-factor, continuous-time process can be backed out of interest rate data.

Here, we extend the work of Stanton (1997) to a multivariate setting and provide for the non-parametric estimation of the drift and volatility functions of multivariate stochastic differential equations. Basically, we use Milshtein's (1978) approximation schemes for writing expectations of functions of the sample path of stochastic differential equations in terms of the drift, volatility and correlation coefficients. If the expectations are known (or, in our case, estimated nonparametrically) and the functions are chosen appropriately, then the approximations can be inverted to recover the drift, volatility and correlation coefficients. In this paper, we apply this technique to the short- and long-end of the term structure for a general two-factor, continuous-time diffusion process for interest rates.

In contrast, the common approach in the literature for investigating multifactor continuoustime interest rate models is to develop implications from the affine class of term structure models. For example, Longstaff and Schwartz (1992) specify preferences and production technologies in such a way that they get closed-form solutions for bond prices and some fixed-income derivatives. Alternatively, other papers write down affine term structure models, with assumptions about the prices of risk, and value securities using no arbitrage. One can view the approach taken in this paper as complementary to this literature. Here, we consider nonlinear specifications for the interest rate process and prices of risk. These general multifactor models then lead to implications for pricing fixed-income securities (albeit without closed forms). The novel aspect of our approach is that rather than choosing the model parameterization out of "thin air", our processes are generated from the data using the Milshtein (1978) approximation schemes described above. As such, one can consider our model a general, nonlinear version of existing multifactor models, such as Longstaff and Schwartz (1992), with the added benefit that, estimation error aside, the model structure is reasonable. In fact, we show directly how our model relates to the two-factor model of Longstaff and Schwartz (1992).

Our paper provides three contributions to the existing literature. First, in estimating this multi-factor diffusion process, some new empirical facts emerge from the data. Of particular note, while the volatility of interest rates increases in the level of interest rates, it does so primarily for sharply upward sloping term structures. Thus, the results of previous studies, suggesting an almost exponential relation between interest rate volatility and levels, is due to the term structure on average being upward sloping, and is not a general result per se. Moreover, our volatility result holds for both the short- and long-term rates of interest. 
Thus, conditional on particular values of the two factors, such as a high short rate of interest and a negative slope of the term structure, the term structure of interest rate volatilities is generally at a lower level across maturities than implied by previous work.

The second contribution is methodological. In this paper, we provide a way of linking empirical facts and continuous-time modeling techniques so that generating implications for fixed-income pricing is straightforward. Specifically, we use nonparametrically estimated conditional moments of "relevant pricing factors" to build a multifactor continuous-time diffusion process which can be used to price securities. This process can be considered a generalization of the Longstaff and Schwartz (1992) two-factor model. Using this estimated process, we then show how to value fixed-income securities, in conjunction with an estimation procedure for the functional for the market prices of risk. Since the analysis is performed nonparametrically without any priors on the underlying economic structure, the method provides a unique opportunity to study the economic structure's implications for pricing. Of course, ignoring the last twenty-five years of term structure theory and placing more reliance on estimation error may not be a viable alternative on its own. Nevertheless, we view this approach as helpful for understanding the relation between interest rate modeling and fixed-income pricing.

The final contribution of the paper is to apply the above methodology to a particular fixed-income pricing application. In particular, we use our continuous-time model to provide an analysis of term premiums. There is a considerable literature in finance on the shape of the term structure of term premiums. Here, both factors, and the underlying nonlinearities of the model, play an important role in fixed-income pricing and can be directly linked to the results in the literature. This is especially interesting given that some of the data, and the corresponding moments, were not used in estimation of the model.

The paper is organized as follows. In Section 2, we provide some general empirical facts about the conditional means and volatilities of the short- and long-rate of interest. Formal tests suggest strong evidence of multi-factor pricing. Section 3 outlines the technique for estimating the underlying multi-factor continuous-time process, and then applies this method to estimate a general two-factor process for interest rates over the 1983 to 1998 period. Of particular interest, this section contains a discussion of several new stylized facts about the stochastic behavior of interest rates. In Section 4, we link our model of interest rate behavior with a generalized version of the popular Longstaff and Schwartz (1992) model, and show how our estimated model can be used to capture an existing stylized fact about interest rates and bond returns across maturities. Section 5 makes some concluding remarks. 


\section{The Stochastic Behavior of Interest Rates: Some Ev- idence}

In this section, we provide some preliminary evidence for the behavior of interest rates across various points of the yield curve. Specifically, under the assumption that there are two interest-rate dependent state variables, and that these variables are spanned by the short rate of interest and the slope of the term structure, we document conditional means and volatilities of changes in the 6-month through 5-year rates of interest. The results are generated nonparametrically, and thus impose no structure on the underlying functional forms for the term structure of interest rates.

\subsection{Data Description}

Daily values for constant maturity treasury yields on the 3-year, 5-year and 10-year U.S. government bond were collected from Datastream over the period January 1983 to December 1998. In addition, 3-month, 6-month and 1-year T-bill rates were obtained from the same source, and converted to annualized yields. This provides us with roughly 4,000 daily observations.

The post-1982 period was chosen because there is considerable evidence that the period prior to 1983 came from a different regime (see, for example, Huizinga and Mishkin (1984), Sanders and Unal (1988), Klemkosky and Pilotte (1992), and Torous and Ball (1995)). In particular, these researchers argue that the October 1979 change in Federal Reserve operating policy led to a once-and-for-all shift in the behavior of the short term riskless rate. Since the Federal Reserve experiment ended in November 1982, it is fairly standard to treat only the post late 1982 period as stationary.

In estimating the conditional distribution of the term structure of interest rates, we employ two conditioning factors. These factors are the short rate of interest - defined here as the 3-month yield - and the slope of the term structure — defined as the spread between the 10-year and 3-month yield. These variables are chosen to coincide with interest rate variables used in other studies (see Litterman and Scheinkman (1991) and Chan, Karolyi, Longstaff and Sanders (1992), among others). Figure 1 graphs the time series of both the short rate and spread. Over the 1983 to 1998 period, the short rate ranges from $3.00 \%$ to $11.50 \%$, while the spread varies from $-0.51 \%$ to $3.50 \%$. There are several distinct periods of low and high interest rates, as well as spread ranges. Since the correlation between the 
short rate and spread is -0.40 , there exists the potential for the two variables combined to possess information in addition to a single factor.

Figure 2 presents a scatter plot of the short rate and term structure slope. Of particular importance to estimating the conditional distribution of interest rates is the availability of the conditioning data. Figure 2 shows that there are two holes in the data ranges, namely at low short rates (i.e., from 3-5\%) and low spreads (i.e., from -0.5-2\%), and at high short rates (i.e., from $9.5-11.5 \%$ ) and low spreads (i.e., from -0.5-1.5\%). This means that the researcher should be cautious in interpreting the implied distribution of interest rates conditional on these values for the short rate and spread.

\subsection{The Conditional Distribution of Interest Rates: A First Look}

In order to understand the stochastic properties of interest rates, consider conditioning the data on four possible states: (i) high level (i.e., of the short rate)/high slope, (ii) high level/low slope, (ii) low level/low slope, and (iv) low level/high slope. In a generalized method of moments framework, the moment conditions are ${ }^{2}$ :

$$
E\left(\begin{array}{c}
\left(\Delta i_{t, t+1}^{\tau}-\mu_{h r: h s}^{\tau}\right) \times I_{t, h r: h s} \\
\left(\Delta i_{t, t+1}^{\tau}-\mu_{h r: l s}^{\tau}\right) \times I_{t, h r: l s} \\
\left(\Delta i_{t, t+1}^{\tau}-\mu_{l r: l s}^{\tau}\right) \times I_{t, l r: l s} \\
\left(\Delta i_{t, t+1}^{\tau}-\mu_{l r: h s}^{\tau}\right) \times I_{t, l r: h s} \\
{\left[\left(\Delta i_{t, t+1}^{\tau}-\mu_{h r: h s}^{\tau}\right)^{2}-\sigma_{h r: h s}^{\tau}{ }^{2}\right] \times I_{t, h r: h s}} \\
{\left[\left(\Delta i_{t, t+1}^{\tau}-\mu_{h r: l s}^{\tau}\right)^{2}-\sigma_{h r: l s}^{\tau}{ }^{2}\right] \times I_{t, h r: l s}} \\
{\left[\left(\Delta i_{t, t+1}^{\tau}-\mu_{l r: l s}^{\tau}\right)^{2}-{\sigma_{l r: l s}^{\tau}}^{2}\right] \times I_{t, l r: l s}} \\
{\left[\left(\Delta i_{t, t+1}^{\tau}-\mu_{l r: h s}^{\tau}\right)^{2}-{\sigma_{l r: h s}^{\tau}}^{\tau}\right] \times I_{t, l r: h s}}
\end{array}\right)=0
$$

where $\Delta i_{t, t+1}^{\tau}$ is the change in the $\tau$-period interest rate from $t$ to $t+1, \mu_{. \mid}^{\tau}$. is the mean change in rates conditional on one of the four states occurring, $\sigma_{. \mid}^{\tau}$. is the volatility of the change in rates conditional on these states, and $I_{t, \mid} \cdot=1$ if $[\cdot \mid \cdot]$ occurs, zero otherwise. These moments, $\mu^{\tau}$ and $\sigma^{\tau}$, thus represent coarse estimates of the underlying conditional moments of the distribution of interest rates.

These moment conditions allow us to test a variety of restrictions. First, are $\sigma_{h r: h s}^{\tau}=\sigma_{h r: l s}^{\tau}$ and $\sigma_{l r: h s}^{\tau}=\sigma_{l r: l s}^{\tau}$ ? That is, does the slope of the term structure help explain volatility at various interest rate levels? Second, similarly, with respect to the mean, are $\mu_{h r: h s}^{\tau}=\mu_{h r: l s}^{\tau}$

\footnotetext{
${ }^{2}$ We define a low (high) level or spread as one that lies below (above) its unconditional mean. Here, this mean is being treated as a known constant, though, of course, it is estimated via the data.
} 
and $\mu_{l r: h s}^{\tau}=\mu_{l r: l s}^{\tau}$ ? Table 1 provides estimates of $\mu_{\cdot \mid \text {. }}^{\tau}$ and $\sigma_{\cdot \mid \cdot}^{\tau}$, and the corresponding test statistics. Note that the framework allows for autocorrelation and heteroskedasticity in the underlying squared interest rate series when calculating the variance-covariance matrix of the estimates. Further, the cross-correlation between the volatility estimates is taken into account in deriving the test statistics.

Several facts emerge from Table 1. First, as documented by others (e.g., Chan, Karolyi, Longstaff and Sanders (1992), and Ait-Sahalia (1996a)), interest rate volatility is increasing in the short rate of interest. Of some interest here, this result holds across the yield curve. That is, conditional on either a low or high slope, volatility is higher for the 6-month, 1-year, 3-year and 5-year rates at higher levels of the short rate. Second, the slope also plays an important role in determining interest rate volatility. In particular, at high levels of interest rates, the volatility of interest rates across maturities is much higher at steeper slopes. For example, the 6-month and 5-year volatilities rise from 6.23 and 6.89 to 8.28 and 8.43 basis points, respectively. Formal tests of the hypothesis $\sigma_{h r: h s}^{\tau}=\sigma_{h r: l s}^{\tau}$ provide $1 \%$ level rejections at each of the maturities. There is some evidence in the literature that expected returns on bonds are higher for steeper term structures (see, for example, Fama (1986) and Boudoukh, Richardson, Smith and Whitelaw (1999a,1999b)), which may provide a link to the volatility result here. Third, the effect of the slope is most important at high interest rate levels. At low short rate levels, though the volatility at low slopes is less than that at high slopes, the effect is much less pronounced. This is confirmed by the fact that a number of the p-values are no longer significant at conventional levels for the test of the hypothesis, $\sigma_{l r: h s}^{\tau}=\sigma_{l r: l s}^{\tau}$. Fourth, the conditional means, though not in general reliably estimated, are consistent with existing results in the literature (e.g., Chan, Karolyi, Longstaff and Sanders (1992), AitSahalia (1996a), and Stanton (1997)). That is, at low levels of interest rates, the mean tends to be greater than at high interest rates, which can be explained by mean reversion. However, the table also provides an interesting new result, namely that the effect of the slope is of higher magnitude than the level. Further, low slopes tend to be associated with negative changes in rates, while high slopes are linked to positive interest rate changes.

To understand the joint properties of interest rates, Table 1 presents the average correlation between the 6-month through 5-year yields, conditional on the four possible states. Conditional on a particular interest rate level, there is little difference between the correlations across interest rates, for low versus high term structure slopes. In contrast, for a given term structure slope, low interest rate levels tend to be associated with lower correlations. For example, conditional on a steep term structure, the correlation is 0.87 and 0.79 at high 
levels and low levels, respectively.

The results of Table 1 suggest a complex variance-covariance structure for changes in the term structure of interest rates, conditional on the current level and slope of the term structure. Below, we take a closer look at this structure by estimating the conditional distribution between interest rate changes and the level and slope.

\subsection{The Conditional Distribution of Interest Rates: A Closer Look}

We employ a kernel estimation procedure for estimating the relation between interest rate changes and components of the term-structure of interest rates. ${ }^{3}$ Kernel estimation is a nonparametric method for estimating the joint density of a set of random variables. Specifically, given a time series $\Delta i_{t, t+1}^{\tau}, i_{t}^{r}$ and $i_{t}^{s}$ (where $i^{r}$ is the level of interest rates, and $i^{s}$ is the slope), generated from an unknown density $f\left(\Delta i^{\tau}, i^{r}, i^{s}\right)$, then a kernel estimator of this density is

$$
\hat{f}\left(\Delta i^{\tau}, i^{r}, i^{s}\right)=\frac{1}{T h^{m}} \sum_{t=1}^{T} K\left(\frac{\left(\Delta i^{\tau}, i^{r}, i^{s}\right)-\left(\Delta i_{t, t+1}^{\tau}, i_{t}^{r}, i_{t}^{s}\right)}{h}\right),
$$

where $K(\cdot)$ is a suitable kernel function and $h$ is the window width or smoothing parameter. This fixed window width estimator is often called the Parzen estimator. The density at any point is estimated as the average of densities centered at the actual data points. The further away a data point is from the estimation point, the less it contributes to the estimated density. Consequently, the estimated density is highest near high concentrations of data points and lowest when observations are sparse.

The econometrician has at his discretion the choice of $K(\cdot)$ and $h$. Results in the kernel estimation literature suggest that any reasonable kernel gives almost optimal results; thus, we employ the commonly used independent multivariate normal kernel. The other parameter, the window width, is chosen based on the dispersion of the observations. For the independent multivariate normal kernel, Scott (1992) suggests the window width,

$$
\hat{h}=k \hat{\sigma}_{i} T^{\frac{-1}{m+4}}
$$

where $\hat{\sigma}_{i}$ is the standard deviation estimate of each variable $z_{i}, T$ is the number of observations, $m$ is the dimension of the variables, and $k$ is a scaling constant often chosen via cross-validation. Here, we employ a cross-validation procedure to find the $k$ which provides the right trade-off between the bias and variance of the errors. Across all the data points,

\footnotetext{
${ }^{3}$ For examples of kernel estimation methods in the empirical asset pricing literature, see Pagan and Hong (1991), Harvey (1991), Ait-Sahalia (1996a) and Boudoukh, Richardson and Whitelaw (1995).
} 
we find the $k$ 's which minimize the mean-squared error between the observed data and the estimated conditional data. This mean-squared error minimization is implemented using a Jackknife-based procedure. In particular, the various implied conditional moments at each data point are estimated using the entire sample, except for the actual data point and its nearest neighbors. ${ }^{4}$ Once the $k$ is chosen, the actual estimation of the conditional distribution of interest rates involves the entire sample, albeit using window widths chosen from partial samples.

Two moments of interest rate changes are particularly interesting to financial economists, namely the mean and volatility. Intuitively, within the kernel estimation procedure, one can view estimates of the volatility and the mean as nonlinearly interpolating between functions of data points (albeit in a multidimensional space). In particular, one can interpret these estimates as a weighted combination of either observed interest rate changes (i.e., for the mean estimate) or squared interest rate changes (i.e., for the volatility estimate). Specifically, it is possible to show that

$$
\begin{aligned}
\hat{\mu}_{\Delta i^{\tau}}\left(i^{r}, i^{s}\right) & =\sum_{t=1}^{T} w_{t}\left(i^{r}, i^{s}\right) \Delta i_{t}^{\tau} \\
{\hat{\sigma^{2}}}_{\Delta i^{\tau}}\left(i^{r}, i^{s}\right) & =\sum_{t=1}^{T} w_{t}\left(i^{r}, i^{s}\right)\left(\Delta i_{t}^{\tau}-\hat{\mu}_{\Delta i^{\tau}}\left(i^{r}, i^{s}\right)\right)^{2}
\end{aligned}
$$

where $w_{t}\left(i^{r}, i^{s}\right)=K\left(\frac{\left(i^{r}, i^{s}\right)-\left(i_{t}^{r}, i_{t}^{s}\right)}{h}\right) / \sum_{t=1}^{T} K\left(\frac{\left(i^{r}, i^{s}\right)-\left(i_{t}^{r}, i_{t}^{s}\right)}{h}\right)$. The weights, $w_{t}\left(i^{r}, i^{s}\right)$, are determined by how close the chosen state, i.e., the particular values of the level and slope, $i^{r}$ and $i^{s}$, is to the observed level and slope of the term structure, $i_{t}^{r}$ and $i_{t}^{s}$.

As an illustration, using equations (3) and (4), Figures 3-4 provide estimates of the mean and volatility of daily changes in the 1-year rate, conditional on the current level of the short rate and the slope of the term structure (i.e., $i_{t}^{r}$ and $i_{t}^{s}$ ). While Figures 3-4 provide estimates for only the 1-year rate, the same effects carry through to the rest of the yield curve and have therefore been omitted for purposes of space. The figure maps these estimates to the relevant range of the data, in particular, for short rates ranging from $3 \%$ to $11 \%$ and slopes ranging from $0.0 \%$ to $3.5 \%$. As seen from Figure 2, there are no observations of both low spreads and either very low or very high interest rate levels. Thus, the parts of the figures relating to these areas of the data should be treated with caution.

\footnotetext{
${ }^{4}$ Due to the serial dependence of the data, we performed the cross-validation omitting 100 observations, i.e., four months in either direction of the particular data point in question. Depending on the moments in question, the optimal $k$ 's range from roughly 2.1 to 11.4 , which implies approximately twice to eleven times the smoothing parameter of Scott's asymptotically optimal implied value.
} 
The cross-validation procedure provides a high smoothing parameter for the estimation of the interest rate's drift. One way to interpret this result is in the context of the existing literature which shows how difficult it is to estimate low fequency phenomena, like mean reversion, in interest rates (see, for example, Bandi (1998), Chapman and Pearson (1998), Jones (1998) and Pritzker (1998)). While the reliability of the estimates in Figure 3 are, therefore, in question, it is interesting to note that the shape of the curve falls in line with standard intuition. At higher rates and lower spreads, the drift is most negative; as the term structure slope increases, and rates are in theory expected to rise, the drift increases to reflect this rise. One notable point is that the drift is everywhere negative. This results from the unfortunate fact that (i) on average, rates drifted down during the sample period, and (ii) the data is oversmoothed to reflect the estimation problems of interest rate drifts.

With respect to Figure 4, while Table 1 gave a rough approximation of these conditional moments, the figure provides a precise functional form (albeit estimated). For example, both pieces of information suggest that volatility for the 1-year rate (and, in fact, all other points on the yield curve) are maximized at high interest rate levels and high slopes. However, the figures shows that the functional relation is relatively smooth and monotonic. That is, rather than being a result about averages, we find that larger squared changes in daily interest rates over the 1983 to 1998 period tend to occur during times of steeper term structure slopes and higher interest rate levels. Furthermore, a 1\% change in the slope is much more important than a $1 \%$ increase in rates at higher interest rate levels. It should be noted though that a $1 \%$ change in the slope is much more dramatic than a corresponding change in rates.

Figures 5 and 6 present cut-throughs of Figure 4 across the term structure at short rates of $8.0 \%$ and $5.5 \%$, respectively. From Figure 2, these levels represent data ranges in which there are many different slopes; thus, conditional on these levels, the estimated relation between the volatility of the 6-month, 1-year, 3-year and 5-year rates as a function of the slope is more reliable. Several observations are in order. First, as seen from the figures, volatility is increasing in the slope for all maturities, though primarily only for steep term structures, i.e., above $2.0 \%$. Second, volatility is also higher at greater magnitudes of the short rate, though this difference is more pronounced at higher slopes. These results suggest that any valuation requiring a volatility estimate of interest rates should be done with caution. For example, estimating volatility when the term structure is flat relative to upward sloping should lead to quite different point estimates. Third, the relation between volatility and the slope is nonlinear, which, as it turns out in Section 3.3, will lead to a nonlinear continuous-time diffusion process. This feature can be potentially important as most of the multifactor, term 
structure pricing models are derived from the affine class.

Alternatively, Figures 7 and 8 provide cut-throughs of Figure 4 across the term structure at slopes of $2.75 \%$ and $1.00 \%$, respectively. These slopes represent data ranges in which there are a number of observations of the interest rate level. The figures show that the estimated relation between the volatility of the 6-month, 1-year, and especially the 3-year and 5-year rates as a function of the level depends dramatically on the slope of the term structure. For

example, the volatility of the 3- and 5-year interest rate change is almost flat over levels of $3.0 \%$ to $8.5 \%$ at low slopes, whereas it increases 2 basis points daily (or approximately 31 basis points on a 250 trading day scale) at high slopes. Similarly, even at the short end of the yield curve, the increase in volatility is 4 basis points daily versus 1.0 basis points at high versus low slopes, respectively.

\section{Estimation of a Continuous-Time Multi-factor Dif- fusion Process}

The results of Section 2 suggest that the distribution of changes in the term structure of interest rates depends on at least two factors. As proxies for the information content of these factors, we used the level and slope of the term structure, as measured respectively by the short rate of interest (i.e., the 3-month rate) and the spread between the long (i.e., the 10-year rate) and short rate. In particular, Figures 3-8 provide functional forms for important moments of the conditional distribution of future interest rate changes, conditional on current information about the interest rate factors. Given the importance of continuoustime mathematics in the fixed income area, the question arises as to how these results can be interpreted in a continuous-time setting. In particular,

- How can these results for discretely sampled data be made to coincide with a continuoustime process?

- What do these results imply for the specification of the drift and diffusion of the twofactor process for interest rates?

- How can this two-factor process then be used to generate implications for valuing fixed-income contingent claims?

Over the past several years, there has been a flurry of research on the estimation of continuous-time interest rate models. The primary reason for this explosion in the literature 
is the predominance of continuous-time mathematics in the fixed-income area. Using data on bond prices, and explicit theoretical pricing models (e.g., Cox, Ingersoll and Ross (1985)), Brown and Dybvig (1989), Pearson and Sun (1994), Gibbons and Ramaswamy (1994) and Dai and Singleton (1997) all estimate parameters of the underlying interest-rate process in a fashion consistent with the underlying continuous-time model. Recently, researchers have taken to more direct examinations of the interest-rate process. For example, Chan, Karolyi, Longstaff and Sanders (1992) examine a continuous-time single-factor model of interest rates by empirically investigating its discrete-time counterpart. However, this type of approximation is difficult to interpret, at least formally, in a continuous-time setting.

As a result, a new literature has emerged which allows estimation and inference of fairly general continuous-time diffusion processes using discretely sampled data. By employing the infinitesimal generators of the underlying continuous-time diffusion processes, Hansen and Scheinkman (1995) and Conley, Hansen, Luttmer and Scheinkman (1995) construct moment conditions which make the investigation of continuous-time models possible with discrete time data. In a nonparametric framework, Ait-Sahalia (1996a,b) develops a procedure for estimating the underlying process for interest rates using discrete data by choosing a model for the drift of interest rates and then nonparametrically estimating its diffusion function. As an alternative method, Stanton (1997) employs approximations to the true drift and diffusion of the underlying process, and then nonparametrically estimates these approximation terms to back out the continuous-time process (see also Bandi (1998), Chapman and Pearson (1998) and Pritzker (1998)). The advantage of this approach is twofold: (i) similar to the other procedures, the data need only be observed at discrete time intervals, and (ii) the drift and diffusion are unspecified, and thus may be highly nonlinear in the state variable.

In this section, we extend the work of Stanton (1997) to a multivariate setting and provide for the non-parametric estimation of the drift and volatility functions of multivariate stochastic differential equations. Similar to Stanton (1997), we use Milshtein's (1978) approximation schemes for writing expectations of functions of the sample path of stochastic differential equations in terms of the drift and volatility coefficients. If the expectations are known (albeit estimated nonparametrically in this paper) and the functions are chosen appropriately, then the approximations can be inverted to recover the drift and volatility coefficients. 


\subsection{Drift, Diffusion and Correlation Approximations}

Assume that no arbitrage opportunities exist, and that bond prices are functions of two state variables, the values of which can always be inverted from the current level, $R_{t}$, and a second state variable, $S_{t}$. Assume that these variables follow the (jointly) Markov diffusion process

$$
\begin{aligned}
d R_{t} & =\mu_{R}\left(R_{t}, S_{t}\right) d t+\sigma_{R}\left(R_{t}, S_{t}\right) d Z_{t}^{R} \\
d S_{t} & =\mu_{S}\left(R_{t}, S_{t}\right) d t+\sigma_{S}\left(R_{t}, S_{t}\right) d Z_{t}^{S},
\end{aligned}
$$

where the drift, volatility and correlation coefficients (i.e., the correlation between $Z^{R}$ and $\left.Z^{S}\right)$ all depend on $R_{t}$ and $S_{t}$. Define the vector $X_{t}=\left(R_{t}, S_{t}\right)$.

Under suitable restrictions on $\mu, \sigma$, and a function $f$, we can write the conditional expectation $E_{t}\left[f\left(X_{t+\Delta}\right)\right]$ in the form of a Taylor series expansion, ${ }^{5}$

$$
E_{t}\left[f\left(X_{t+\Delta}\right)\right]=f\left(X_{t}\right)+\mathcal{L} f\left(X_{t}\right) \Delta+\frac{1}{2} \mathcal{L}^{2} f\left(X_{t}\right) \Delta^{2}+\ldots+\frac{1}{n !} \mathcal{L}^{n} f\left(X_{t}\right) \Delta^{n}+O\left(\Delta^{n+1}\right),
$$

where $\mathcal{L}$ is the infinitesimal generator of the multivariate process $\left\{X_{t}\right\}$ (see Oksendal (1985) and Hansen and Scheinkman (1995)), defined by

$$
\mathcal{L} f\left(X_{t}\right)=\left(\frac{\partial f\left(X_{t}\right)}{\partial X_{t}}\right) \mu_{X}\left(X_{t}\right)+\frac{1}{2} \operatorname{trace}\left[\Sigma\left(X_{t}\right)\left(\frac{\partial^{2} f\left(X_{t}\right)}{\partial X_{t} \partial X_{t}^{\prime}}\right)\right]
$$

where

$$
\Sigma\left(X_{t}\right)=\left(\begin{array}{cc}
\sigma_{R}^{2}\left(R_{t}, S_{t}\right) & \rho\left(R_{t}, S_{t}\right) \sigma_{R}\left(R_{t}, S_{t}\right) \sigma_{S}\left(R_{t}, S_{t}\right) \\
\rho\left(R_{t}, S_{t}\right) \sigma_{R}\left(R_{t}, S_{t}\right) \sigma_{S}\left(R_{t}, S_{t}\right) & \sigma_{S}^{2}\left(R_{t}, S_{t}\right)
\end{array}\right) .
$$

Equation 7 can be used to construct numerical approximations to $E_{t}\left[f\left(X_{t+\Delta}\right)\right]$ in the form of a Taylor series expansion, given known functions $\mu_{R}, \mu_{S}, \rho, \sigma_{R}$ and $\sigma_{S}$ (see, for example, Milshtein (1978)). Alternatively, given an appropriately chosen set of functions

\footnotetext{
${ }^{5}$ For a discussion see, for example, Hille and Phillips (1957), Chapter [11]. Milshtein $(1974,1978)$ gives examples of conditions under which this expansion is valid, involving boundedness of the functions $\mu, \sigma, f$ and their derivatives. There are some stationary processes for which this expansion does not hold for the functions $f$ that we shall be considering, including processes such as

$$
d x=\mu d t+x^{3} d Z
$$

which exhibit "volatility induced stationary" (see Conley, Hansen, Luttmer and Scheinkman (1995)). However, any process for which the first order Taylor series expansion fails to hold (for linear $f$ ) will also fail if we try to use the usual numerical simulation methods (e.g. Euler discretization). This severely limits their usefulness in practice.
} 
$f(\cdot)$ and nonparametric estimates of $E_{t}\left[f\left(X_{t+\Delta}\right)\right]$, we can use Equation 7 to construct approximations to the drift, volatility and correlation coefficients (i.e., $\mu_{R}, \mu_{S}, \rho, \sigma_{R}$ and $\sigma_{S}$ ) of the underlying multifactor, continuous-time diffusion process. The nice feature of this method is that the functional forms for $\mu_{R}, \mu_{S}, \rho, \sigma_{R}$ and $\sigma_{S}$ are quite general, and can be estimated nonparametrically from the underlying data. Rearranging Equation 7, and using a time step of length $i \Delta(i=1,2, \ldots)$, we obtain

$$
\begin{aligned}
\widehat{E}^{i}\left(X_{t}\right) & \equiv \frac{1}{i \Delta} E_{t}\left[f\left(X_{t+i \Delta}\right)-f\left(X_{t}\right)\right] \\
& =\mathcal{L} f\left(X_{t}\right)+\frac{1}{2} \mathcal{L}^{2} f\left(X_{t}\right)(i \Delta)+\ldots+\frac{1}{n !} \mathcal{L}^{n} f\left(X_{t}\right)(i \Delta)^{n-1}+O\left(\Delta^{n}\right)
\end{aligned}
$$

From Equation 8, each of the $\widehat{E}^{i}$ is a first order approximation to $\mathcal{L} f$,

$$
\widehat{E}^{i}\left(X_{t}\right)=\mathcal{L} f\left(X_{t}\right)+O(\Delta) .
$$

Now consider forming linear combinations of these approximations, $\sum_{i=1}^{N} \alpha_{i} \widehat{E}^{i}\left(X_{t}\right)$. That is, from Equation 8,

$$
\sum_{i=1}^{N} \alpha_{i} \widehat{E}^{i}\left(X_{t}\right)=\left[\sum_{i=1}^{N} \alpha_{i}\right] \mathcal{L} f\left(X_{t}\right)+\frac{1}{2}\left[\sum_{i=1}^{N} \alpha_{i} i\right] \mathcal{L}^{2} f\left(X_{t}\right) \Delta+\frac{1}{6}\left[\sum_{i=1}^{N} \alpha_{i} i^{2}\right] \mathcal{L}^{3} f\left(X_{t}\right) \Delta^{2}+\ldots
$$

Can we choose the $\alpha_{i}$ so that this linear combination is an approximation to $\mathcal{L} f$ of order $N ?$

For the combination to be an approximation to $\mathcal{L} f$, we require first that the weights $\alpha_{1}, \alpha_{2}, \ldots, \alpha_{N}$ sum to 1 . Furthermore, from Equation 9, in order to eliminate the first order error term, the weights must satisfy the equation

$$
\sum_{i=1}^{N} \alpha_{i} i=0
$$

More generally, in order to eliminate the $n$th order error term $(n \leq N-1)$, the weights must satisfy the equation,

$$
\sum_{i=1}^{N} \alpha_{i} i^{n}=0
$$

We can write this set of restrictions more compactly in matrix form as

$$
\left(\begin{array}{ccccc}
1 & 1 & 1 & \cdots & 1 \\
1 & 2 & 3 & \cdots & N \\
1 & 4 & 9 & \cdots & N^{2} \\
\vdots & \vdots & \vdots & \ddots & \vdots \\
1 & 2^{N-1} & 3^{N-1} & \cdots & N^{N-1}
\end{array}\right) \alpha \equiv V \alpha=\left(\begin{array}{c}
1 \\
0 \\
0 \\
\vdots \\
0
\end{array}\right) .
$$


The matrix $V$ is called a Vandermonde matrix, and is invertible for any value of $N$. We can thus obtain $\alpha$ by calculating

$$
\alpha=V^{-1}\left(\begin{array}{c}
1 \\
0 \\
\vdots \\
0 .
\end{array}\right) .
$$

For example, for $N=3$, we obtain

$$
\begin{aligned}
\alpha & =\left(\begin{array}{lll}
1 & 1 & 1 \\
1 & 2 & 3 \\
1 & 4 & 9
\end{array}\right)^{-1}\left(\begin{array}{l}
1 \\
0 \\
0
\end{array}\right), \\
& =\left(\begin{array}{c}
3 \\
-3 \\
1
\end{array}\right) .
\end{aligned}
$$

Substituting $\alpha$ into Equation 9, and using Equation 8, we get the following third order approximation of the infinitesimal generator of the process $\left\{X_{t}\right\}$ :

$$
\begin{gathered}
\mathcal{L} f\left(X_{t}\right)=\frac{1}{6 \Delta}\left[18 E_{t}\left(f\left(X_{t+\Delta}\right)-f\left(X_{t}\right)\right)-9 E_{t}\left(f\left(X_{t+2 \Delta}\right)-f\left(X_{t}\right)\right)+2 E_{t}\left(f\left(X_{t+3 \Delta}\right)-f\left(X_{t}\right)\right)\right] \\
+O\left(\Delta^{3}\right) .
\end{gathered}
$$

To approximate a particular function $g(x)$, we now need merely to find a specific function $f$ satisfying

$$
\mathcal{L} f(x)=g(x) .
$$

For our purposes, consider the functions

$$
\begin{aligned}
f_{(1)}(R) & \equiv R-R_{t}, \\
f_{(2)}(S) & \equiv S-S_{t}, \\
f_{(3)}(R) & \equiv\left(R-R_{t}\right)^{2}, \\
f_{(4)}(S) & \equiv\left(S-S_{t}\right)^{2}, \\
f_{(5)}(R, S) & \equiv\left(R-R_{t}\right)\left(S-S_{t}\right) .
\end{aligned}
$$

From the definition of $\mathcal{L}$, we have

$$
\mathcal{L} f_{(1)}(R)=\mu_{R}(R, S),
$$




$$
\begin{aligned}
\mathcal{L} f_{(2)}(S) & =\mu_{S}(R, S), \\
\mathcal{L} f_{(3)}(R) & =2\left(R-R_{t}\right) \mu_{R}(R, S)+\sigma_{R}^{2}(R, S), \\
\mathcal{L} f_{(4)}(S) & =2\left(S-S_{t}\right) \mu_{S}(R, S)+\sigma_{S}^{2}(R, S), \\
\mathcal{L} f_{(5)}(R, S) & =\left(S-S_{t}\right) \mu_{R}(R, S)+\left(R-R_{t}\right) \mu_{S}(R, S)+\rho(R, S) \sigma_{R}(R, S) \sigma_{S}(R, S) .
\end{aligned}
$$

Evaluating these at $R=R_{t}, S=S_{t}$, we obtain

$$
\begin{aligned}
\mathcal{L} f_{(1)}\left(R_{t}\right) & =\mu_{R}\left(R_{t}, S_{t}\right), \\
\mathcal{L} f_{(2)}\left(S_{t}\right) & =\mu_{S}\left(R_{t}, S_{t}\right), \\
\mathcal{L} f_{(3)}\left(R_{t}\right) & =\sigma_{R}^{2}\left(R_{t}, S_{t}\right), \\
\mathcal{L} f_{(4)}\left(S_{t}\right) & =\sigma_{S}^{2}\left(R_{t}, S_{t}\right), \\
\mathcal{L} f_{(5)}\left(R_{t}, S_{t}\right) & =\rho\left(R_{t}, S_{t}\right) \sigma_{R}\left(R_{t}, S_{t}\right) \sigma_{S}\left(R_{t}, S_{t}\right) .
\end{aligned}
$$

Using each of these functions in turn as the function $f$ above, we can generate approximations to $\mu_{R}, \mu_{S}, \sigma_{R}, \sigma_{S}$ and $\rho$ respectively. For example, the third order approximations (taking square roots for $\sigma_{R}$ and $\sigma_{S}$ ) are

$$
\begin{aligned}
\mu_{R}\left(R_{t}, S_{t}\right)= & \frac{1}{6 \Delta}\left[18 E_{t}\left(R_{t+\Delta}-R_{t}\right)-9 E_{t}\left(R_{t+2 \Delta}-R_{t}\right)+2 E_{t}\left(R_{t+3 \Delta}-R_{t}\right)\right] \\
& +O\left(\Delta^{3}\right), \\
\mu_{S}\left(R_{t}, S_{t}\right)= & \frac{1}{6 \Delta}\left[18 E_{t}\left(S_{t+\Delta}-S_{t}\right)-9 E_{t}\left(S_{t+2 \Delta}-S_{t}\right)+2 E_{t}\left(S_{t+3 \Delta}-S_{t}\right)\right] \\
& +O\left(\Delta^{3}\right), \\
\sigma_{R}\left(R_{t}, S_{t}\right)= & \sqrt{\frac{1}{6 \Delta}\left(18 E_{t}\left[\left(R_{t+\Delta}-R_{t}\right)^{2}\right]-9 E_{t}\left[\left(R_{t+2 \Delta}-R_{t}\right)^{2}\right]+2 E_{t}\left[\left(R_{t+3 \Delta}-R_{t}\right)^{2}\right]\right)} \\
\sigma_{S}\left(R_{t}, S_{t}\right)= & \sqrt{\frac{1}{6 \Delta}\left(18 E_{t}\left[\left(S_{t+\Delta}-S_{t}\right)^{2}\right]-9 E_{t}\left[\left(S_{t+2 \Delta}-S_{t}\right)^{2}\right]+2 E_{t}\left[\left(S_{t+3 \Delta}-S_{t}\right)^{2}\right]\right)} \\
\sigma_{R S}\left(R_{t}, S_{t}\right)= & \frac{1}{6 \Delta}\left(18 E_{t}\left[\left(R_{t+\Delta}-R_{t}\right)\left(S_{t+\Delta}-S_{t}\right)\right]-9 E_{t}\left[\left(R_{t+2 \Delta}-R_{t}\right)\left(S_{t+2 \Delta}-S_{t}\right)\right]\right. \\
& \left.\quad+2 E_{t}\left[\left(R_{t+3 \Delta}-R_{t}\right)\left(S_{t+3 \Delta}-S_{t}\right)\right]\right) .
\end{aligned}
$$

The approximations of the drift, volatility and correlation coefficients are written in terms of the true first, second and cross moments of multiperiod changes in the two state variables. If the two-factor assumption is appropriate, and a large stationary time series is available, then these conditional moments can be estimated using appropriate nonparametric methods. In this paper, we estimate the moments using multivariate density estimation, with appropriately chosen factors as the conditioning variables. All that is required is that 
these factors span the same space as the true state variables. ${ }^{6}$ The results for daily changes were provided in Section 2. Equation 13 shows that these estimates are an important part of the approximations to the underlying continuous-time dynamics. By adding multiperiod extensions of these nonparametric estimated conditional moments, we can estimate the drift, volatility and correlation coefficients of the multifactor process described by Equations 5 and 6.

Figure 9 provides the first, second and third order approximations to the diffusion of the short rate against the short rate level and the slope of the term structure. ${ }^{7}$ The most notable result is that a first order approximation works well; thus, one can consider the theoretical results of this section as a justification for discretization methods currently used in the literature, e.g., Chan, Karolyi, Longstaff and Sanders (1992). The description of interest rate behavior given in Section 2, therefore, carries through to the continuous-time setting. The question then is what does Figure 9, and more generally the rest of the estimated process, mean for fixed-income pricing?

\section{A Generalized Longstaff and Schwartz (1992) Model}

Longstaff and Schwartz (1992) provide a two-factor general equilibrium model of the term structure. Their model is one of the more popular versions within the affine class of models for describing the yield curve (see also Cox, Ingersoll and Ross (1985), Chen and Scott (1993) and Duffie and Kan (1996)). In the Longstaff and Schwartz setting, all fixed-income instruments are functions of two fundamental factors, the instantaneous interest rate and its volatility. These factors follow diffusion processes, which in turn lead to a fundamental valuation condition for the price of any bond, or bond derivative. As an alternative, here we also present a two-factor continuous-time model for interest rates. The results of Section 2 suggest that the affine class may be too restrictive.

While our results shed valuable light on the factors driving interest rate movements, however, there are potential problems in using this specification to price interest rate contingent claims. A general specification for $R_{t}$ and $S_{t}$ (and the associated prices of risk) may allow

\footnotetext{
${ }^{6}$ See Duffie and Kan (1996) for a discussion of the conditions under which this is possible (in a linear setting).

${ }^{7}$ Figures showing the various approximations to the drift of the short rate, the drift and diffusion of the slope, and the correlation between the short rate and the slope are available upon request.
} 
arbitrage opportunities if either of these state variables is a known function of an asset price. ${ }^{8}$ Of course, this point is true of all previous estimations of continuous-time processes to the extent that they use a priced proxy as the instantaneous rate. If we are willing to assume that we have the right factors, then there is no problem in an asymptotic sense. That is, since we are estimating these processes nonparametrically, as the sample size gets larger, our estimates will converge to the true functions, which are automatically arbitrage-free (if the economy is). Nevertheless, this is of little consolation if we are trying to use the estimated functions to price assets.

To get around this problem, we need to write the model in a form in which neither state variable is an asset price or a function of asset prices. In this paper, we follow convention by using the observable 3 -month yield as a proxy for the instantaneous rate, $R_{t}$. Furthermore, suppose that the mapping from $(R, S)$ to $\left(R, \sigma_{R}\right)$ is invertible, ${ }^{9}$ so we can write asset prices as a function of $R$ and $\sigma_{R}$, instead of $R$ and $S .{ }^{10}$ Since $\sigma_{R}$ is not an asset price, using this variable avoids the inconsistency problem.

Specifically, suppose that the true model governing interest rate movements is a generalization of the two factor Longstaff and Schwartz (1992) model,

$$
\begin{aligned}
d R_{t} & =\mu_{R}(R, \sigma) d t+\sigma d Z_{1}, \\
d \sigma_{t} & =\mu_{\sigma}(R, \sigma) d t+\rho(R, \sigma) s(R, \sigma) d Z_{1}+\sqrt{1-\rho^{2}} s d Z_{2}
\end{aligned}
$$

where $d Z_{1} d Z_{2}=0 .{ }^{11}$ In vector terms,

$$
d\left(R_{t}, \sigma_{t}\right)=M d t+\theta d Z
$$

\footnotetext{
${ }^{8}$ See, for example, Duffie, Ma and Wong (1995). The problem is that, given such a model, we can price any bond, and are thus able to calculate what the state variable "ought" to be. Without imposing any restrictions on the assumed dynamics for $R_{t}$ and $S_{t}$, there is no guarantee that we will get back to the same value of the state variable that we started with.

${ }^{9}$ That is, for a given value of $R_{t}$, the volatility, $\sigma_{R}$ is monotonic in the spread, $S$. This is the case in most existing multifactor interest rate models, including, for example all affine models, such as Longstaff and Schwartz (1992).

${ }^{10}$ This follows by writing

$$
V(R, S)=V\left(R, S\left(R, \sigma_{R}\right)\right) \equiv U\left(R, \sigma_{R}\right)
$$

${ }^{11}$ This specification is the most convenient to deal with, since we now have orthogonal noise terms. The correlation between the diffusion terms is $\rho$, and the overall variance of $\sigma$ is $s^{2} d t$.
} 
where

$$
\begin{aligned}
M & \equiv\left(\begin{array}{l}
\mu_{R} \\
\mu_{\sigma}
\end{array}\right), \\
\theta & \equiv\left(\begin{array}{cc}
\sigma & 0 \\
\rho s & \sqrt{1-\rho^{2}} s
\end{array}\right) .
\end{aligned}
$$

Asset prices, and hence the slope of the term structure, can be written as some function of the short rate and instantaneous short rate volatility, $S(R, \sigma)$.

From Equations 14 and 15, how do we estimate the underlying processes for $R$ and $\sigma$ given the estimation results of Section 3? Although the short rate volatility, $\sigma$, is not directly observable, it is possible to estimate this process. Specifically, using Ito's Lemma, together with estimates for $\mu_{R}, \sigma_{R}, \mu_{S}, \sigma_{S}$ and $\rho$, it is possible to write

$d \sigma_{t}=\sigma_{R} d R_{t}+\sigma_{S} d S_{t}+\frac{1}{2}\left[\sigma_{R R} \sigma^{2}\left(R_{t}, S_{t}\right)+\sigma_{S S} \sigma_{S}^{2}\left(R_{t}, S_{t}\right)+2 \sigma_{R S} \sigma\left(R_{t}, S_{t}\right) \sigma_{S}\left(R_{t}, S_{t}\right) \rho\left(R_{t}, S_{t}\right)\right] d t$.

Given this equation, and the assumption that the function $S(R, \sigma)$ is invertible, the dynamics of $\sigma_{t}$ can be written as a function of the current level of $R$ and $\sigma$ in a straightforward way.

This procedure requires estimation of a matrix of second derivatives. Although there are well-known problems in estimating higher-order derivatives using kernel density estimation techniques, it is possible to link the results of Section 2 and 3 to this generalized Longstaff and Schwartz (1992) model. In particular, using estimates of the second derivatives (not shown), several facts emerge. First, due to the small magnitudes of the estimated drifts of the state variables $R$ and $S$, the drift of $\sigma$ depends primarily on the second order terms. Consequently, the importance of the second factor (the slope) is determined by how much the sensitivity of short rate volatility to this factor changes relative to the changes in the sensitivty to the first factor (the level). From Figure 9, it is clear that the second derivatives are somewhat unstable, especially in the $R$ dimension. Nevertheless, the general pattern is that volatility increases at a slower rate for high slopes and levels. Consequently, for high volatilities, the drift of volatility is negative, generating mean reversion. The effect of the second factor is to reinforce this phenomenon. Second, the diffusion of $\sigma$ is determined by the sensitivities of short rate volatility to the two factors and the magnitudes of the volatilities of the factors. Based on the estimates of the volatilites and derivatives, the slope has the dominant influence on this effect. In particular, the volatility of $\sigma$ is high for upward sloping term structures, which also correspond to states with high short rate volatility. Moreover, sensitivity of this diffusion to the two factors is larger in the slope direction than in the level direction. 
As an alternative to the above method, we can estimate an implied series for $\sigma$ by assuming that the function $S(R, \sigma)$ is invertible, i.e., that we can equivalently write the model in the form

$$
\begin{gathered}
d R_{t}=\mu_{R}\left(R_{t}, S_{t}\right) d t+\sigma\left(R_{t}, S_{t}\right) d Z_{1}^{*} \\
d S_{t}=\mu_{S}\left(R_{t}, S_{t}\right) d t+\sigma_{S}\left(R_{t}, S_{t}\right) d Z_{2}^{*}
\end{gathered}
$$

where $Z_{1}^{*}$ and $Z_{2}^{*}$ may be correlated. To estimate the function $\sigma(R, S)$, we apply the methodology described in Section 3.1 to the function $f_{(3)}(R, S) \equiv\left(R-R_{t}\right)^{2}$. Applying the estimated function to each observed $(R, S)$ pair in turn yields a series for the volatility $\sigma$, which we can then use in estimating the generalized Longstaff and Schwartz (1992) model given in Equations 14 and 15. ${ }^{12}$ This procedure is in stark contrast to that of Longstaff and Schwartz (1992), and others, who approximate the dynamics of the volatility factor as a Generalized Autoregressive Conditional Heteroskedasticity (GARCH) process. The GARCH process is not compatible with the underlying dynamics of their continuous-time model; here, the estimation is based on approximation schemes to the diffusion process and is internally consistent. Due to the difficulties in estimating derivatives, we choose this second approach to estimate the continuous-time process. ${ }^{13}$

\subsection{A General Two Factor Diffusion Process: Empirical Results}

Figures 11-14 show approximations to the drift and diffusion coefficients for the generalized Longstaff and Schwartz (1992) process as a function of the two factors, the instantaneous short rate and its volatility. It is important to point out that there is little available data at low short rates/high volatilities and high short rates/low volatilities, which corresponds to the earlier comment about interest rates and spreads (see Figure 10). Therefore, results in these regions need to be treated cautiously. With respect to the interest rate drift, Figure 11 shows a very similar figure to that of Figure 3. Here, interest rate volatility is proxying for the slope of the term structure, or vice versa. Again, the figure is dramatically smoothed through cross-validation due to the inability of the estimation method (or for that matter any method) to uncover reliable functional forms for mean reversion in interest rates.

\footnotetext{
${ }^{12}$ Although the use of an estimated series for $\sigma$ rather than the true series may not be the most efficient approach, this procedure is consistent. That is, the problem will disappear as the sample size becomes large, and our pointwise estimates of $\sigma$ converge to the true values.

${ }^{13}$ Though the first approach provides similar results to the second approach, the functional forms underlying the second method are more smooth and thus more suitable for analysis.
} 
More interesting, Figures 12 and 13 provide the estimates of the continuous-time process for the second interest factor, namely its volatility. Several observations are in order. First, there is estimated mean-reversion in volatility; at low (high) levels of volatility, volatility tends to drift upward (downward). The effect of the level of interest rates on this relation appears minimal. Second, and perhaps most important, there is clear evidence that the diffusion of the volatility process is increasing in the level of volatility, yet is affected by the level of interest rates only marginally. Moreover, volatility's effect is nonlinear in that it takes affect only at higher levels. This finding suggests extreme caution should be applied when inputting interest rate volatility into derivative pricing models. Most of our models take the relation between the level and volatility for granted; however, with increases from $3 \%$ to $11 \%$ in the interest rate level, only mild increases in volatility are being reported for both volatility's drift and diffusion. On the other hand, changes in the volatility level of much smaller magnitudes have a much larger impact on the volatility process. This finding links the spread result documented earlier in the paper to a second factor, namely the volatility of the instantaneous rate.

As the final piece of the multifactor process for interest rates, Figure 14 graphs a third order approximation of the correlation coefficient between the short rate and the volatility, given values of the two factors. Taken at face value, the results suggest a complex variance-covariance matrix between these series in continuous-time. In particular, while the correlation decreases in the volatility for most interest rate levels, there appears to be some nonmonotonicity across the level itself. Why is correlation falling as volatility increases? Perhaps, high volatility, just like the corresponding high term structure slope, is associated with aggregate economic phenomena that are less related to the level of interest rates. Given that interest rates are driven by two relatively independent economic factors, namely expectations about both real rates and inflation, this argument seems reasonable. It remains an open question, however, what the exact relation is between Figure 14 and these economic factors.

\subsection{Valuation of Fixed-Income Contingent Claims}

Given the interest rate model described in equation (15), we can write the price of an interest rate contingent claim as $V(r, \sigma, t)$, depending only on the current values of the two 
state variables plus time. Then, by Ito's Lemma,

$$
\frac{d V(r, \sigma, t)}{V(r, \sigma, t)}=m(r, \sigma, t) d t+s_{1}(r, \sigma, t) d Z_{1}+s_{2}(r, \sigma, t) d Z_{2}
$$

where

$$
\begin{aligned}
m(r, \sigma, t) V & =V_{t}+\mu_{r}(r, \sigma) V_{R}+\mu_{\sigma}(r, \sigma) V_{\sigma}+\frac{1}{2} \operatorname{trace}\left[\theta^{T} \nabla^{2} V(r, \sigma) \theta\right], \\
& =V_{t}+\mu_{r}(r, \sigma) V_{r}+\mu_{\sigma}(r, \sigma) V_{\sigma}+\frac{1}{2} \sigma^{2} V_{r r}+\frac{1}{2} s^{2} V_{\sigma \sigma}+\rho \sigma s V_{r \sigma}, \\
s_{1}(r, \sigma, t) V & =\sigma V_{r}+\rho s V_{\sigma}, \\
s_{2}(r, \sigma, t) V & =\sqrt{1-\rho^{2}} s V_{\sigma} .
\end{aligned}
$$

The volatility of the asset, $\sigma_{V}$, is given by

$$
\begin{aligned}
\sigma_{V} V & =\sqrt{\left(\sigma V_{r}+\rho s V_{\sigma}\right)^{2}+\left(1-\rho^{2}\right) s^{2} V_{\sigma}^{2}} \\
& =\sqrt{\sigma^{2} V_{r}^{2}+2 \rho \sigma s V_{r} V_{\sigma}+s^{2} V_{\sigma}^{2}} .
\end{aligned}
$$

With a one factor interest rate model, to prevent arbitrage, the risk premium on any asset must be proportional to its standard deviation. ${ }^{14}$ Similarly, with two factors, absence of arbitrage requires the excess return on an asset to be a linear combination of its exposure to the two sources of risk. Thus, if the asset pays out dividends at rate $d$, we can write

$$
m=r-\frac{d}{V}+\lambda_{r}(r, \sigma) \frac{V_{r}}{V}+\lambda_{\sigma}(r, \sigma) \frac{V_{\sigma}}{V},
$$

where $\lambda_{r}$ and $\lambda_{\sigma}$ are the prices of short rate risk and volatility risk respectively. Substituting equation (18) into equation (17), and simplifying, leads to a partial differential equation that must be satisfied by any interest rate contingent claim, assuming the usual technical smoothness and integrability conditions (see, for example, Duffie (1988)),

$$
\frac{1}{2} \sigma^{2} V_{r r}+\left[\mu_{r}-\lambda_{r}\right] V_{r}+\frac{1}{2} s^{2} V_{\sigma \sigma}+\left[\mu_{\sigma}-\lambda_{\sigma}\right] V_{\sigma}+\rho \sigma s V_{r \sigma}+V_{t}-r V+d=0,
$$

subject to appropriate boundary conditions. To price interest rate dependent assets, we need to know not only the processes governing movements in $r$ and $\sigma$, but also the prices of risk, $\lambda_{r}$ and $\lambda_{\sigma}$.

Given estimates for the process governing movements in $r$ and $\sigma$, and also for the functions $\lambda_{r}$ and $\lambda_{\sigma}$, we can value interest rate dependent assets in one of two ways. The first is to solve

\footnotetext{
${ }^{14}$ Suppose this did not hold for two risky assets. We could then create a riskless portfolio of these two assets with a return strictly greater than $r$, leading to an arbitrage opportunity (see Ingersoll(1987)).
} 
equation (19) numerically using a method such as the Hopscotch method of Gourlay and McKee (1977). The second is to use the fact that we can write the solution to equation (19) in the form of an expectation. Specifically, we can write $V$, the value of an asset which pays out cash flows at a (possibly path dependent) rate $C_{t}$, in the form

$$
V_{t}=E\left[\int_{t}^{T} e^{-\int_{t}^{s}\left(\widehat{r}_{u}\right) d u} C_{s} d s\right],
$$

where $\widehat{r}$ follows the "risk adjusted" process,

$$
\begin{aligned}
d \widehat{r}_{\tau} & =\left[\mu_{r}\left(\widehat{r}_{\tau}, \widehat{\sigma}_{\tau}\right)-\lambda_{r}\left(\widehat{r}_{\tau}, \widehat{\sigma}_{\tau}\right)\right] d \tau+\widehat{\sigma}_{\tau} d Z_{1}, \\
d \widehat{\sigma}_{\tau} & =\left[\mu_{\sigma}\left(\widehat{r}_{\tau}, \widehat{\sigma}_{\tau}\right)-\lambda_{\sigma}\left(\widehat{r}_{\tau}, \widehat{\sigma}_{\tau}\right)\right] d \tau+\rho s\left(\widehat{r}_{t} a u, \widehat{\sigma}_{\tau}\right) d Z_{1}+\sqrt{1-\rho^{2}} s d Z_{2},
\end{aligned}
$$

for all $\tau>t$, and where

$$
\begin{aligned}
& \widehat{r}_{t}=r_{t}, \\
& \widehat{\sigma}_{t}=\sigma_{t} .
\end{aligned}
$$

This says that the value of the asset equals the expected sum of discounted cash flows paid over the life of the asset, except that it substitutes the risk adjusted process $(\widehat{r}, \widehat{\sigma})$ for the true process $(r, \sigma)$.

This representation leads directly to a valuation algorithm based on Monte Carlo simulation. For a given starting value of $\left(r_{t}, \sigma_{t}\right)$, simulate a number of paths for $\widehat{r}$ and $\widehat{\sigma}$ using equations (21) and (22). Along each path, calculate the cash flows $C_{t}$, and discount these back along the path followed by the instantaneous riskless rate, $\widehat{r}_{t}$. The average of the sum of these values taken over all simulated paths is an approximation to the expectation in equation (20), and hence to the security value, $V_{t}$. The more paths simulated, the closer the approximation.

\subsubsection{Estimating the Prices of Risk}

Recall that to price interest rate dependent assets, we need to know not only the processes governing movements in $r$ and $\sigma$, but also the prices of risk, $\lambda_{r}$ and $\lambda_{\sigma}$. Equation (18) gives an expression for these functions in terms of the partial derivatives $V_{r}$ and $V_{\sigma}$, which could be used to estimate the prices of risk, given estimates of these derivatives for two different assets, plus estimates of the excess return for each asset. As mentioned above, it is difficult to estimate derivatives precisely using nonparametric density estimation. Therefore, instead 
of following this route, we shall avoid directly estimating the partial derivatives, $V_{r}$ and $V_{\sigma}$, by considering the instantaneous covariances between the asset return and changes in the interest rate/volatility, $c_{V r}$ and $c_{V \sigma}$. From equations (14), (15) and (16) (after a little simplification),

$$
\left(\begin{array}{c}
c_{V r} \\
c_{V \sigma}
\end{array}\right) \equiv\left(\begin{array}{c}
d V d r / V d t \\
d V d \sigma / V d t
\end{array}\right)=\left(\begin{array}{cc}
\sigma^{2} & \rho \sigma s \\
\rho \sigma s & s^{2}
\end{array}\right)\left(\begin{array}{c}
V_{r} / V \\
V_{\sigma} / V
\end{array}\right) .
$$

This can be inverted, as long as $|\rho|<1$, to obtain

$$
\begin{aligned}
\left(\begin{array}{c}
V_{r} / V \\
V_{\sigma} / V
\end{array}\right) & =\left(\begin{array}{cc}
\sigma^{2} & \rho \sigma s \\
\rho \sigma s & s^{2}
\end{array}\right)^{-1}\left(\begin{array}{c}
c_{V r} \\
c_{V \sigma}
\end{array}\right), \\
& =\frac{1}{1-\rho^{2}}\left(\begin{array}{cc}
1 / \sigma^{2} & -\rho / \sigma s \\
-\rho / \sigma s & 1 / s^{2}
\end{array}\right)\left(\begin{array}{l}
c_{V r} \\
c_{V \sigma}
\end{array}\right) .
\end{aligned}
$$

To preclude arbitrage, the excess return on the asset must also be expressible as a linear combination of $c_{V r}$ and $c_{V \sigma}$,

$$
m=r-\frac{d}{V}+\lambda^{*}{ }_{r}(r, \sigma) c_{V r}+\lambda^{*}{ }_{\sigma}(r, \sigma) c_{V \sigma} .
$$

Given two different interest rate dependent assets, we can estimate the instantaneous covariances for each in the same way as we estimated $\rho(r, \sigma)$ above. We can also estimate the excess return for each asset, $m_{i}(r, \sigma)-r$ as a function of the two state variables. The two excess return can be expressed in the form

$$
\left(\begin{array}{c}
m_{1}-r \\
m_{2}-r
\end{array}\right)=\left(\begin{array}{cc}
c_{V r}^{1} & c_{V \sigma}^{1} \\
c_{V r}^{2} & c_{V \sigma}^{2}
\end{array}\right)\left(\begin{array}{c}
\lambda_{r}^{*} \\
\lambda^{*}{ }_{\sigma}
\end{array}\right)
$$

which can be inverted to yield an estimate of the prices of risk,

$$
\left(\begin{array}{c}
\lambda^{*}{ }_{r} \\
\lambda^{*}{ }_{\sigma}
\end{array}\right)=\left(\begin{array}{ll}
c_{V r}^{1} & c_{V \sigma}^{1} \\
c_{V r}^{2} & c_{V \sigma}^{2}
\end{array}\right)^{-1}\left(\begin{array}{l}
m_{1}-r \\
m_{2}-r
\end{array}\right)
$$

Finally, for estimates of the more standard representation of the prices of risk, $\lambda_{r}$ and $\lambda_{\sigma}$, equate equations (18) and (24), using equation (23), to obtain

$$
\left(\begin{array}{l}
\lambda_{r} \\
\lambda_{\sigma}
\end{array}\right)=\left(\begin{array}{cc}
\sigma^{2} & \rho \sigma s \\
\rho \sigma s & s^{2}
\end{array}\right)\left(\begin{array}{c}
\lambda^{*}{ }_{r} \\
\lambda^{*}{ }_{\sigma}
\end{array}\right)
$$


Figures 15 and 16 provide estimates of the prices of risk for the instantaneous rate and volatility in terms of the current levels of these variables. While the researcher needs to be a little cautious in interpreting the results due to the range of observed data, several observations are in order. First, the price of short rate risk tends to be negative. One way of interpreting this result is that, for a given volatility and interest rate level, the short rate needs to be risk-adjusted upward. The main effect of a negative price is to make term premiums positive and upward sloping. Second, the price of short rate risk is more negative at lower rates; in contrast, the effect of volatility has an ambiguous effect on this price. Third, the price of volatility risk seems to be relatively flat for most values of the interest rate level and its volatility. Only at higher volatilities does the price of risk vary, with low (high) rates imposing a high (low) price.

\subsection{The Term Structure of Term Premiums}

Figures 11-16 provide a complete description of the underlying processes for interest rates and interest rate risk. Using these estimates, and the valuation theory outlined in Section 4.2 , we can begin to address the following question:

What are the implications of the empirical facts, such as the especially high volatility at steep slopes, for expected returns on bonds?

This is an important question, which, to date, is unanswered in the literature due to the multifactor, nonlinear nature of these facts. Below, we provide a first pass at understanding these implications by focusing on the term structure of term premiums.

There is substantial support in the literature for time-varying risk premia on bonds. For example, Shiller, Campbell and Schoenholtz (1983), Fama (1984, 1986), Keim and Stambaugh (1986), Fama and Bliss (1987), Stambaugh (1988), Campbell and Shiller (1991), Klemkosky and Pilotte (1992) and Engle and Ng (1993) all report evidence that the risk premia on bonds of various maturities are predictable. Moreover, a common conditioning variable is the slope of the term structure, since it can be shown to embed expectations about future rates as well as risk premiums on bonds. In this paper, we have transformed a two-factor world with the level and slope into a generalized Longstaff and Schwartz (1992) model.

Figure 17 documents the term structure of term premiums at two different short rates as implied by the pricing model described earlier in this section. The short rates were chosen to coincide with the available data and the earlier Figures 5 and 6 . Specifically, we report 
the ex ante excess return on the 6-month, 1-year, 3-year and 5-year par bonds, as a function of the short rate's volatility. Several observations are in order. First, these term premiums vary with both the level and the volatility. Recall from equation 18 that these excess returns are determined by two components: (i) the prices of risk, and (ii) the sensitivities of the bond prices to the interest rate factors. The former component is dominated by the short rate (see Figures 15 and 16), while the latter component is related to interest rate volatility, which is related mostly to the slope (see Figure 9).

Second, the predictability appears highly nonlinear, with both the level of the short rate and the degree of interest volatility playing important roles. For example, given high levels of volatility, the term structure of term premiums is at a higher level at rates of $5.5 \%$ than at rates of $8 \%$. In contrast, at low levels of volatility, the term structure of term premiums is similar, irrespective of the interest rate level. Third, and most interesting, is that the overall effect of multiple factors is consistent with the stylized facts in the term premium literature (see, for example, Fama (1986), Fama and Bliss (1987) and Boudoukh, Richardson, Smith and Whitelaw (1999a,1999b)). In particular, it is well-documented that there exists a positive relation between the term structure slope and the term structure of expected returns on bonds. Given the link between volatility and the slope, it should not be surprising then that, for low levels of volatility, the term structure of term premiums is relatively flat compared to that for high levels of volatility.

\section{Conclusion}

This paper provides a method for estimating multifactor continuous-time Markov processes. Using Milshtein's (1978) approximation schemes for writing expectations of functions of the sample path of stochastic differential equations in terms of the drift, volatility and correlation coefficients, we provide non-parametric estimation of the drift and diffusion functions of multivariate stochastic differential equations. We apply this technique to the short- and long-end of the term structure for a general two-factor, continuous-time diffusion process for interest rates. In estimating this process, one major result is that the volatility of interest rates is increasing in the level of interest rates, only for sharply, upward sloping term structures. Thus, the result of previous studies, suggesting an almost exponential relation between interest rate volatility and levels, is due to the term structure on average being upward sloping, and is not a general result per se. Moreover, the slope of the term structure, on its own, plays a large role in determining the magnitude of the diffusion coefficient. These 
volatility results hold across maturities, which suggests that a low dimensional system (with nonlinear effects) may be enough to explain the term structure of interest rates.

There are several advantages of the procedure adopted in this paper. First, there is a constant debate between researchers on the relative benefits of using equilibrium versus arbitrage-free models. Here, we circumvent this issue by using actual data to give us the process and corresponding prices of risk. Since the real world coincides with the intersection of equilibrium and arbitrage-free models, our model is automatically consistent. Of course, in a small sample, statistical error will produce estimated functional forms that do not conform. This problem, however, is true of all empirical work. Second, one of the motivations of this paper is to use our estimates of the underlying multifactor continuous-time diffusion process to generate pricing results, which may lead to an explanation of some of the stylized facts in the literature. We show how our results can be interpreted within a generalized Longstaff and Schwartz (1992) framework, that is, one in which the drift and diffusion coefficients of the instantaneous interest rate and volatility are both (nonlinear) functions of the level of interest rates and the volatility. Very preliminary results suggest that this more generalized model will have some success at explaining the term structure of bond premiums in a unified framework. Third, the approach of this paper may be useful in providing forecasts of the conditional distribution of changes in the term structure of interest rates. As a first pass, the model is reasonably adept at replicating some of the important characteristics of the estimated conditional distribution. Due to the Markov property of the model, these forecasts are not limited to the frequency of the observed data. We are currently working on research along these lines. Fourth, and perhaps most important, the pricing of fixed-income derivatives depends crucially on the level of volatility. The results in this paper suggest that volatility depends on both the level and slope of the term structure. This result, coupled with the prices of risk, lead us to believe that the model produced here may have insights into the pricing of these derivatives. We are currently analyzing the implications of these types of models for valuing interest rate caps and floors. 


\section{References}

Ait-Sahalia, Yacine, 1996a, "Nonparametric Pricing of Interest Rate Derivative Securities," Econometrica $64,527-560$.

Ait-Sahalia, Yacine, 1996b, "Testing Continuous-Time Models of the Spot Interest Rate," Review of Financial Studies, 9(2):385-426.

Andersen, T. and J. Lund, 1997, "Estimating continuous time stochastic volatility models of the short term interest rate," Journal of Econometrics 77, 343-377.

Boudoukh, Jacob, Matthew Richardson, Tom Smith and Robert F. Whitelaw, 1999a, "Ex Ante Bond Returns and the Liquidity Preference Hypothesis," Journal of Finance, 54(3):1153-1167.

Boudoukh, Jacob, Matthew Richardson, Tom Smith and Robert F. Whitelaw, 1999b, "Bond Returns and Regime Shifts," working paper, NYU.

Brennan, Michael and Eduardo Schwartz, 1979, "A Continuous Time Approach to the Pricing of Bonds," Journal of Banking and Finance, 3:133-155.

Brenner, Robin, Richard Harjes and Kenneth Kroner, 1996, "Another Look at Models of the ShortTerm Interest Rate," Journal of Financial and Quantitative Analysis, 31(1):85-107.

Brown, Stephen and Phil Dybvig, 1986, "The Empirical Implications of the Cox, Ingersoll Ross Theory of the Term Structure of Interest Rates," Journal of Finance 41, 617-630..

Campbell, John, 1995, "Some Lessons from the Yield Curve," working paper no. 5031, NBER.

Campbell, John and Robert Shiller, 1991, "Yield Spreads and Interest Rate Movements: A Bird's Eye View," Review of Economic Studies 58, 495-514.

Chan, K.C., A. Karolyi, F. Longstaff and A. Sanders, 1992, "An Empirical Comparison of Alternative Models of the Short-Term Interest Rate," Journal of Finance, 47:1209-1227.

Chapman, D. A., and Pearson, N. D., 1998, "Is the Short Rate Drift Actually Nonlinear," forthcoming Journal of Finance.

Chen, Ren-Raw and Louis Scott, 1992, "Pricing Interest rate Options in a Two-Factor CoxIngersoll-Ross Model of the Term Structure," Review of Financial Studies, 5(4):613-636.

Conley, Timothy, Lars Hansen, Erzo Luttmer and Jose Scheinkman, 1995, "Estimating Subordinated Diffusions from Discrete Time Data," The Review of Financial Studies, 10:525-577.

Cox, John C., Jonathan E. Ingersoll, Jr. and Stephen A. Ross, 1985, "A Theory Of The Term Structure Of Interest Rates," Econometrica 53, 385-408.

Dai, Qiang and Kenneth J. Singleton, 1997, "Specification Analysis of Affine Term Structure Models," working paper (Stanford University).

Duffie, Darrell, 1988, Security Markets: Stochastic Models, Academic Press, Boston.

Duffie, Darrell and Rui Kan, 1996, "A yield-factor model of interest rates," Mathematical Finance $6,379-406$.

Duffie, Darrell, J. Ma, and J. Yong, 1995, "Black's consol rate conjecture," Annals of Applied Probability, 5(2):356-382. 
Fama, Eugene F., 1986, "Term Premiums And Default Premiums In Money Markets," Journal of Financial Economics 17, 175-196.

Gibbons, Michael and Krishna Ramaswamy, 1993, "A Test of the Cox, Ingersoll and Ross Model of the Term Structure," Review of Financial Studies 6, 619-658.

Hansen, Lars and Jose Scheinkman, 1995, "Back to the Future: Generating Moment Implications for Continuous Time Markov Processes," Econometrica 63, 767-804.

Heath, David, Robert Jarrow, and Andrew Morton, 1992, "Bond Pricing and the Term Structure of Interest Rates," Econometrica 60, 77-105.

Hille, E. and R. Phillips, 1957, Functional Analysis and Semigroups, American Mathematical Society, Providence, R.I.

Huizinga, John and Frederic S. Mishkin, 1986, "Monetary Policy Regime Shifts and the Unusual Behavior of Real Interest Rates," Carnegie-Rochester Conference on Public Policy, 24, 231-274.

Ingersoll, Jonathan, 1987, Theory of Financial Decision Making, Rowman and Littlefield, Totowa, NJ.

Jones, C. S., 1998, "A Simple Bayesian Method for the Analysis of Diffusion and Jump-Diffusion Processes," working paper, University of Pennsylvania.

Klemkosky, Robert C. and Eugene A. Pilotte, 1992, "Time-Varying Term Premiums On U.S. Treasury Bills And Bonds," Journal of Monetary Economics 30, 87-106.

Litterman, Robert and Jose Scheinkman, 1991, "Common Factors Affecting Bond Returns," Journal of Fixed Income 1, 54-61.

Longstaff, Francis and Eduardo Schwartz, 1992, "Interest rate volatility and the term structure: A two-factor general equilibrium model," Journal of Finance, 47(4):1259-1282.

Milshtein, G.N., 1974, "Approximate Integration of Stochastic Differential Equations," Theory of Probability and Its Applications, 19:557-562.

Milshtein, G.N., 1978, "A Method of Second-Order Accuracy Integration for Stochastic Differential Equations," Theory of Probability and Its Applications, 23:396-401.

Oksendal, B., 1985, Stochastic Differential Equations: An Introduction with Applications, 3rd edition, Springer-Verlag, New York.

Pearson, Neal and Tong-Shen Sun, 1994, "Exploiting the Conditional Density in Estimating the Term Structure: An Application to the Cox, Ingersoll Ross Model," Journal of Finance 49, 12791304.

Pritsker, M., 1998 " Nonparametric Density Estimation of Tests of Continuous Time Interest Rate Models," The Review of Financial Studies, 11:449-487.

Sanders, A.B. and H. Unal, 1988, "On the Intertemporal Stability of the Short Term Rate of Interest," Journal of Financial and Quantitative Analysis 23, 417-423.

Schaefer, Stephen and Eduardo Schwartz, 1984, "A Two-Factor Model of the Term Structure: An Approximate Analytical Solution," Journal of Financial and Quantitative Analysis, 19:413-424. 
Stambaugh, Robert F., 1988, "The Information In Forward Rates: Implications For Models Of The Term Structure," Journal of Financial Economics 21, 41-70.

Stanton, Richard, 1997, "A Nonparametric Model of Term Structure Dynamics and the Market Price of Interest Rate Risk," Journal of Finance 52, 1973-2002.

Torous, Walter and Clifford Ball 1995, "Regime Shifts in Short Term Riskless Interest Rates," working paper (London Business School). 


\section{TABLE 1: Conditional Moments of Daily Interest Rate Changes (basis points)}

\begin{tabular}{|c|c|c|c|c|}
\hline & High Level, High Slope & High Level, Low Slope & Low Level, High Slope & Low Level, Low Slope \\
\hline & 6-month & 6-month & 6-month & 6-month \\
\hline $\begin{array}{l}\text { Mean } \\
\text { (Std. err) }\end{array}$ & $\begin{array}{c}0.008 \\
(0.288)\end{array}$ & $\begin{array}{l}-0.346 \\
(0.222)\end{array}$ & $\begin{array}{c}0.093 \\
(0.152)\end{array}$ & $\begin{array}{l}-0.165 \\
(0.132)\end{array}$ \\
\hline \multirow[t]{3}{*}{$\begin{array}{l}\text { Hypothesis } \\
\chi^{2} \text { Test }\end{array}$} & $\begin{array}{c}(\mathrm{HR}, \mathrm{HS})=(\mathrm{HR}, \mathrm{LS}) 6-\mathrm{mth} \\
0.951(.33 \mathrm{p}-\mathrm{val})\end{array}$ & $\begin{array}{c}(\mathrm{HR}, \mathrm{HS})=(\mathrm{HR}, \mathrm{LS}) 1-\mathrm{yr} \\
1.093(.30 \mathrm{p}-\mathrm{val})\end{array}$ & $\begin{array}{c}(\mathrm{LR}, \mathrm{HS})=(\mathrm{LR}, \mathrm{LS}) 6-\mathrm{mth} \\
1.657(.20 \mathrm{p}-\mathrm{val})\end{array}$ & $\begin{array}{c}(\mathrm{LR}, \mathrm{HS})=(\mathrm{LR}, \mathrm{LS}) 1-\mathrm{yr} \\
1.889(.17 \mathrm{p}-\mathrm{val})\end{array}$ \\
\hline & High Level, High Slope & High Level, Low Slope & Low Level, High Slope & Low Level, Low Slope \\
\hline & 3 -year & 3 -year & 3-year & 3 -year \\
\hline $\begin{array}{l}\text { Mean } \\
\text { (Std. err) }\end{array}$ & $\begin{array}{l}-0.063 \\
(0.296)\end{array}$ & $\begin{array}{l}-0.312 \\
(0.249)\end{array}$ & $\begin{array}{c}0.049 \\
(0.184)\end{array}$ & $\begin{array}{l}-0.217 \\
(0.174)\end{array}$ \\
\hline \multirow[t]{3}{*}{$\begin{array}{l}\text { Hypothesis } \\
\chi^{2} \text { Test }\end{array}$} & $\begin{array}{c}(\mathrm{HR}, \mathrm{HS})=(\mathrm{HR}, \mathrm{LS}) 3-\mathrm{yr} \\
0.415(.52 \mathrm{p}-\mathrm{val})\end{array}$ & $\begin{array}{c}(\mathrm{HR}, \mathrm{HS})=(\mathrm{HR}, \mathrm{LS}) 5-\mathrm{yr} \\
0.087(.77 \mathrm{p}-\mathrm{val})\end{array}$ & $\begin{array}{c}(\mathrm{LR}, \mathrm{HS})=(\mathrm{LR}, \mathrm{LS}) 3-\mathrm{yr} \\
1.104(.29 \mathrm{p}-\mathrm{val})\end{array}$ & $\begin{array}{c}(\mathrm{LR}, \mathrm{HS})=(\mathrm{LR}, \mathrm{LS}) 5-\mathrm{yr} \\
0.962(.33 \mathrm{p}-\mathrm{val})\end{array}$ \\
\hline & High Level, High Slope & High Level, Low Slope & Low Level, High Slope & Low Level, Low Slope \\
\hline & 6-month & 6-month & 6-month & 6-month \\
\hline \multirow[t]{2}{*}{$\begin{array}{l}\text { Vol } \\
\text { (Std. err) } \\
\text { Hypothesis } \\
\chi^{2} \text { Test }\end{array}$} & $\begin{array}{cc}8.275 & 8.685 \\
(0.526) & (0.534) \\
(\mathrm{HR}, \mathrm{HS})=(\mathrm{HR}, \mathrm{LS}) 6-\mathrm{mth} \\
12.160(.00 \mathrm{p} \text {-val })\end{array}$ & $\begin{array}{cc}6.229 & 6.925 \\
(0.247) & (0.292) \\
(\mathrm{HR}, \mathrm{HS})=(\mathrm{HR}, \mathrm{LS}) 1-\mathrm{yr} \\
8.181(.00 \mathrm{p}-\mathrm{val})\end{array}$ & $\begin{array}{cc}5.328 & 5.813 \\
(0.227) & (0.214) \\
(\mathrm{LR}, \mathrm{HS})=(\mathrm{LR}, \mathrm{LS}) & 6-\mathrm{mth} \\
6.772 & (.01 \mathrm{p}-\mathrm{val})\end{array}$ & $\begin{array}{cc}4.480 & 4.996 \\
(0.227) & (0.213) \\
(\mathrm{LR}, \mathrm{HS})=(\mathrm{LR}, \mathrm{LS}) 1-\mathrm{yr} \\
7.048(.01 \mathrm{p}-\mathrm{val})\end{array}$ \\
\hline & 3 -year & 3-year & 3 -year & 3 -year \\
\hline \multirow[t]{3}{*}{$\begin{array}{l}\text { Vol } \\
\text { (Std. err) } \\
\text { Hypothesis } \\
\chi^{2} \text { Test }\end{array}$} & $\begin{array}{lc}8.514 & 8.427 \\
(0.494) & (0.475) \\
(\mathrm{HR}, \mathrm{HS})=(\mathrm{HR}, \mathrm{LS}) 3-\mathrm{yr} \\
\quad 6.888(.01 \mathrm{p}-\mathrm{val})\end{array}$ & $\begin{array}{cc}6.996 & 6.887 \\
(0.288) & (0.273) \\
(\mathrm{HR}, \mathrm{HS})=(\mathrm{HR}, \mathrm{LS}) 5-\mathrm{yr} \\
7.729(.01 \mathrm{p}-\mathrm{val})\end{array}$ & $\begin{array}{lc}6.476 & 6.480 \\
(0.205) & (0.208) \\
(\mathrm{LR}, \mathrm{HS})=(\mathrm{LR}, \mathrm{LS}) 3-\mathrm{yr} \\
3.680(.06 \mathrm{p}-\mathrm{val})\end{array}$ & $\begin{array}{cc}5.890 & 5.984 \\
(0.215) & (0.209) \\
(\mathrm{LR}, \mathrm{HS}) & (\mathrm{LR}, \mathrm{LS}) 5-\mathrm{yr} \\
2.680 & (.10 \mathrm{p}-\mathrm{val})\end{array}$ \\
\hline & \multicolumn{4}{|c|}{ Average Correlation } \\
\hline & 0.870 & 0.842 & 0.787 & 0.794 \\
\hline
\end{tabular}

Table 1 presents summary statistics for daily changes in the 6-month, 1-year, 3year, and 5-year yields on U.S. government securities over the 1983 to 1998 period. Specifically, the table provides the mean, volatility, and cross-correlation of these series, conditional on whether the level of the short rate and slope of the term structure are either low or high. A Wald test that the conditional moments are equal is also provided for the mean and volatility of these series. 


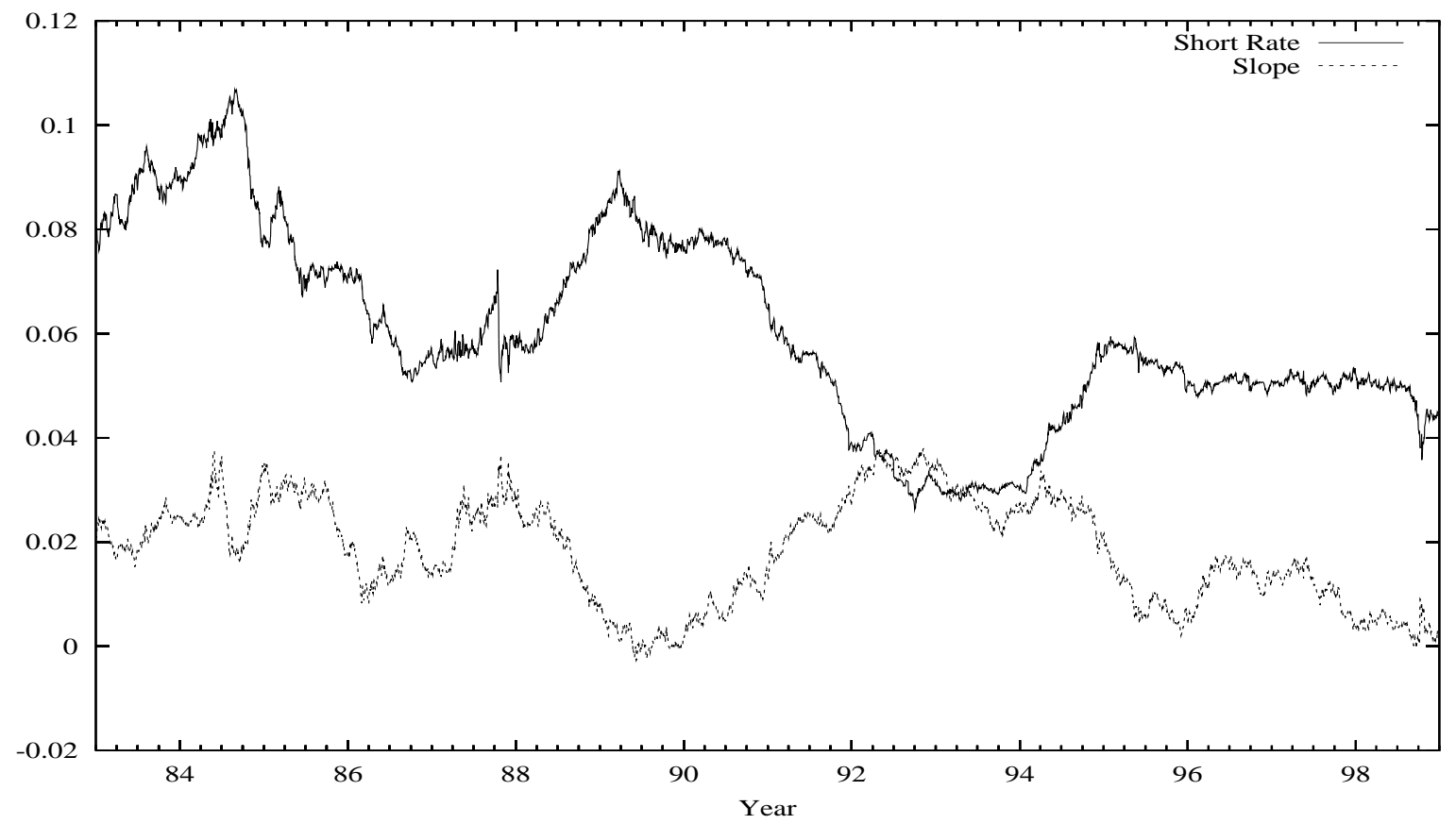

Figure 1: Time series plot of the 3-month rate and term structure slope (i.e., the spread between the 10-year and 3-month rate) over the 1983-1996 period.

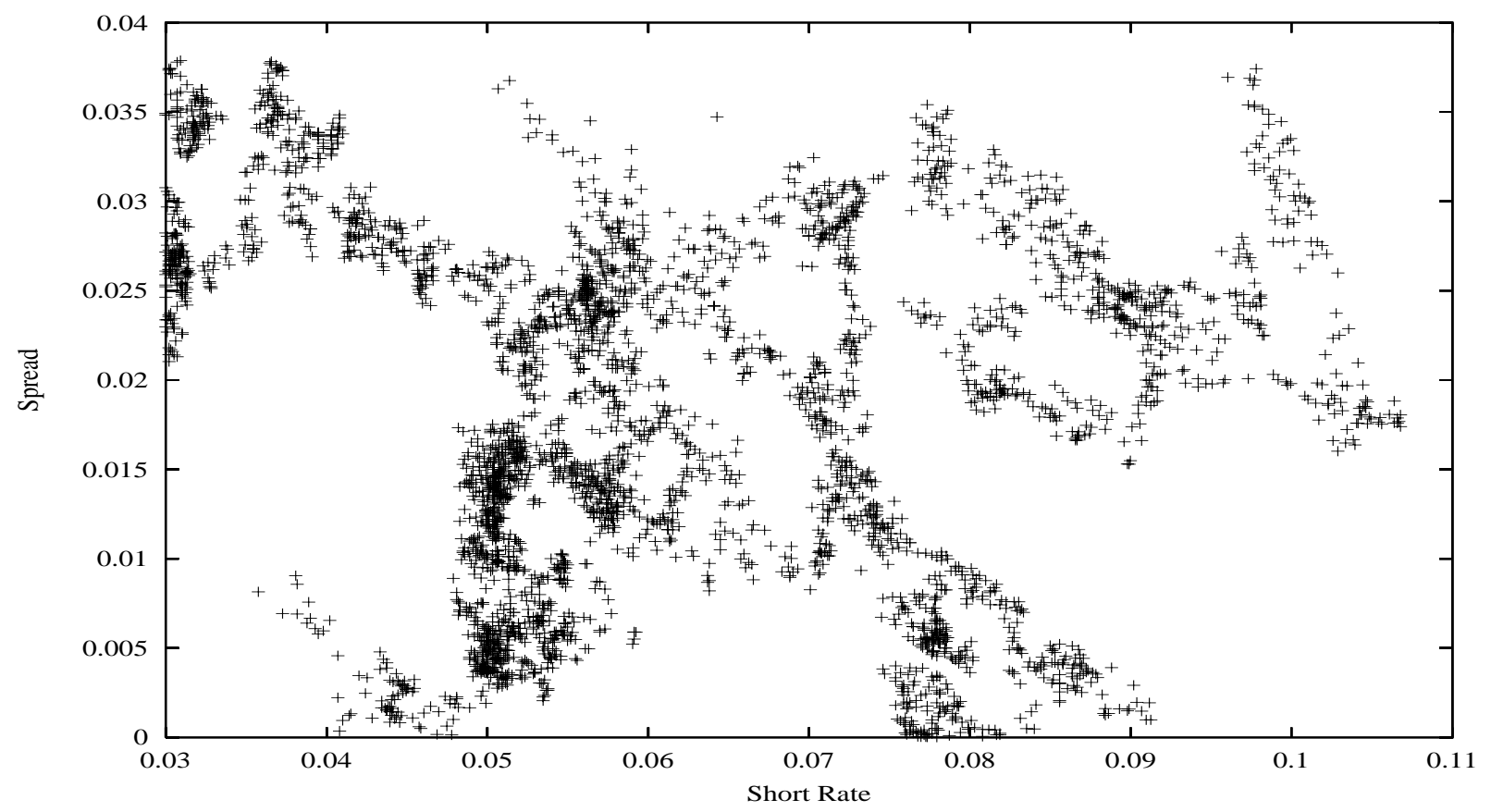

Figure 2: Scatter plot of the 3-month rate vs. the term structure slope over the 1983-1996 period. 


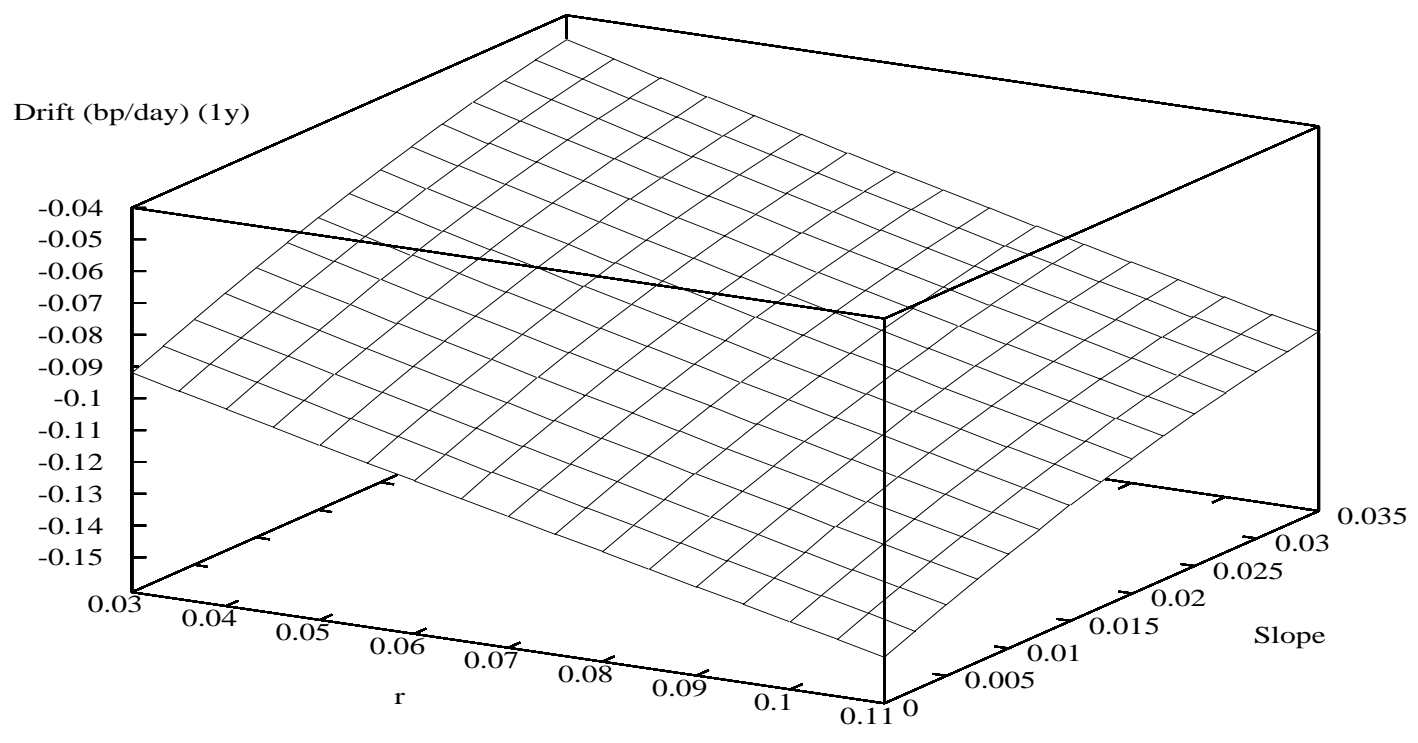

Figure 3: The mean of the daily change in the 1-year yield (in basis points), conditional on the short rate and the slope of term structure.

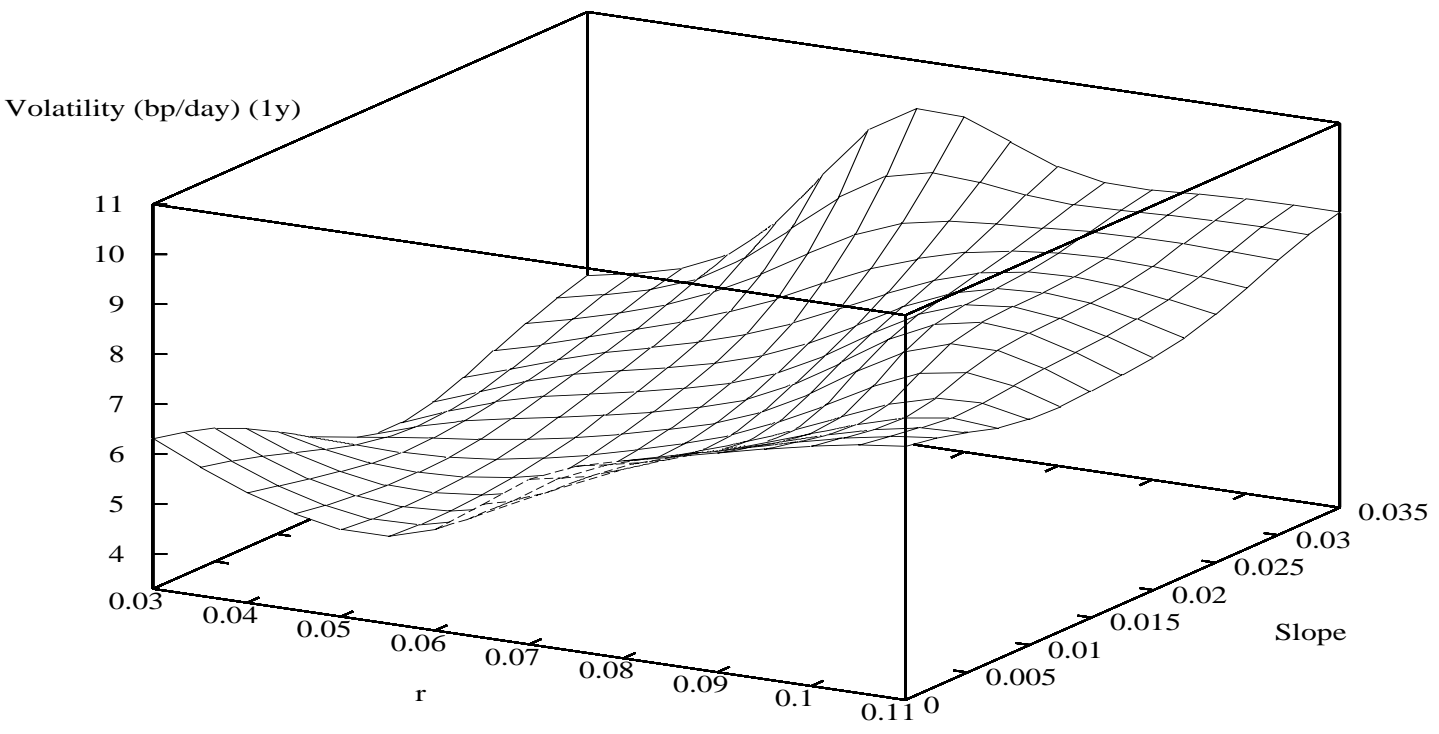

Figure 4: The volatility of the daily change in the 1-year yield (in basis points), conditional on the short rate and the slope of term structure. 


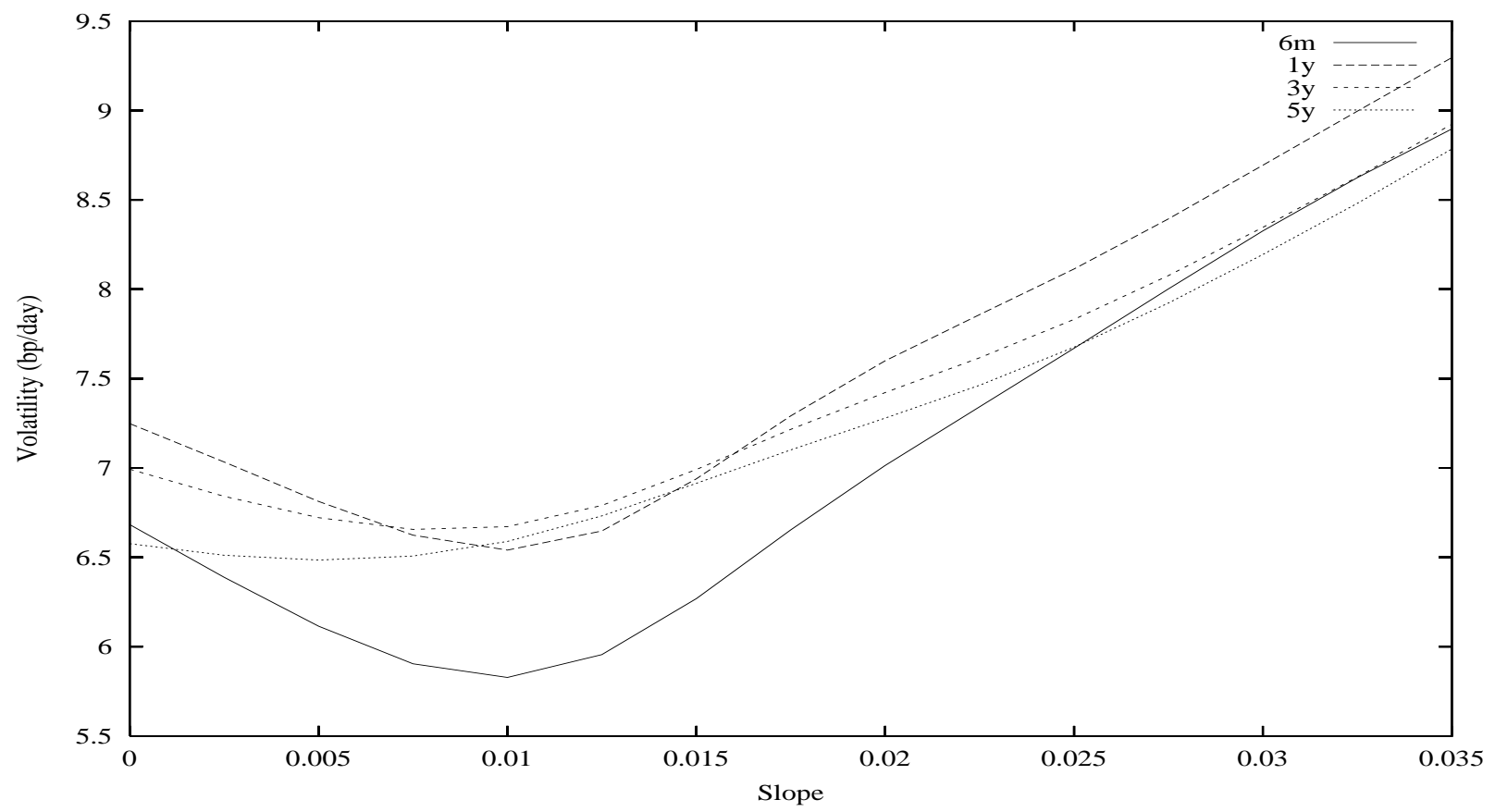

Figure 5: The volatility of the daily change in yields vs. the spread, with the short rate fixed at $8 \%$.

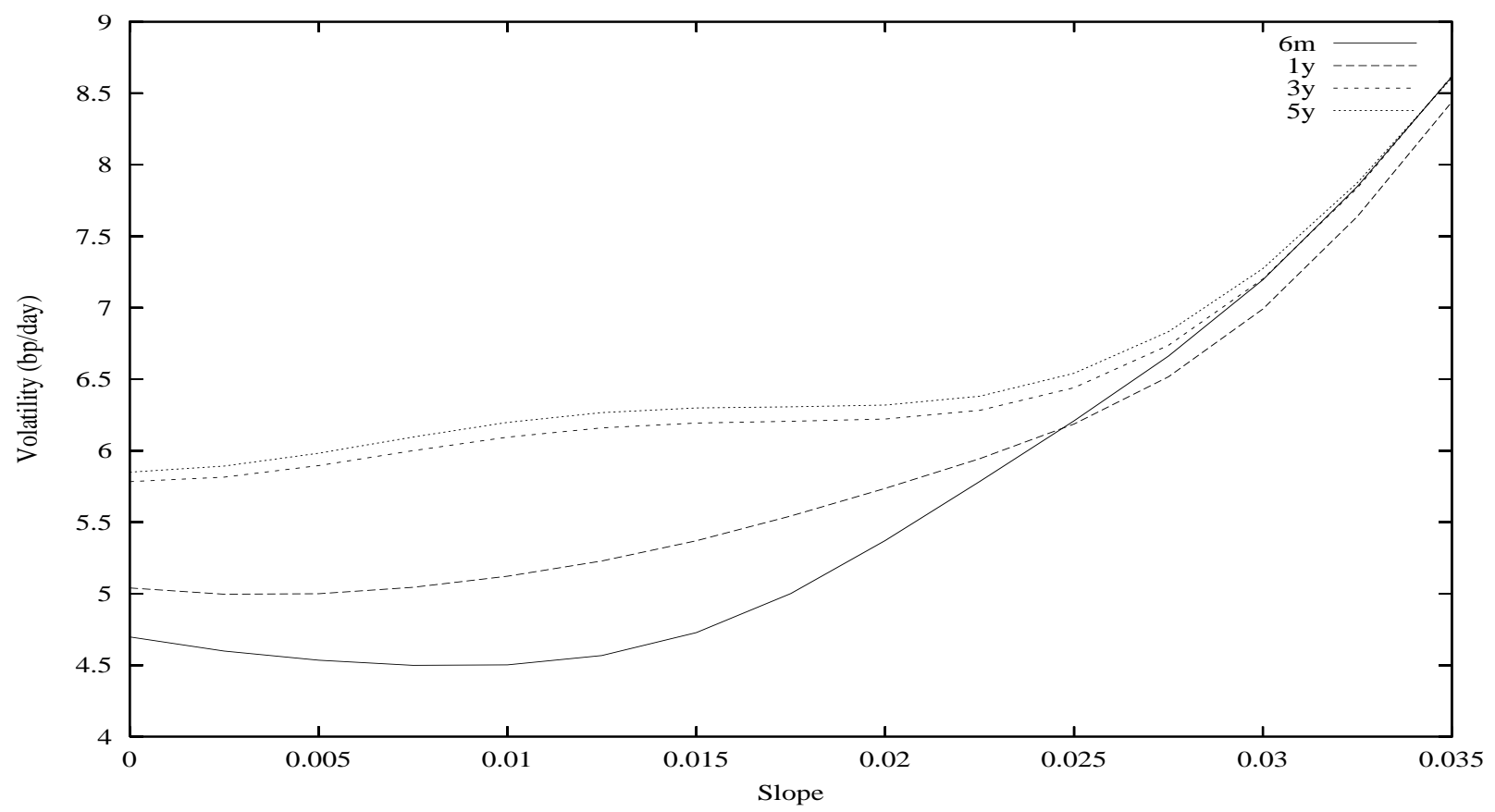

Figure 6: The volatility of the daily change in yields vs. the spread, with the short rate fixed at $5.5 \%$. 


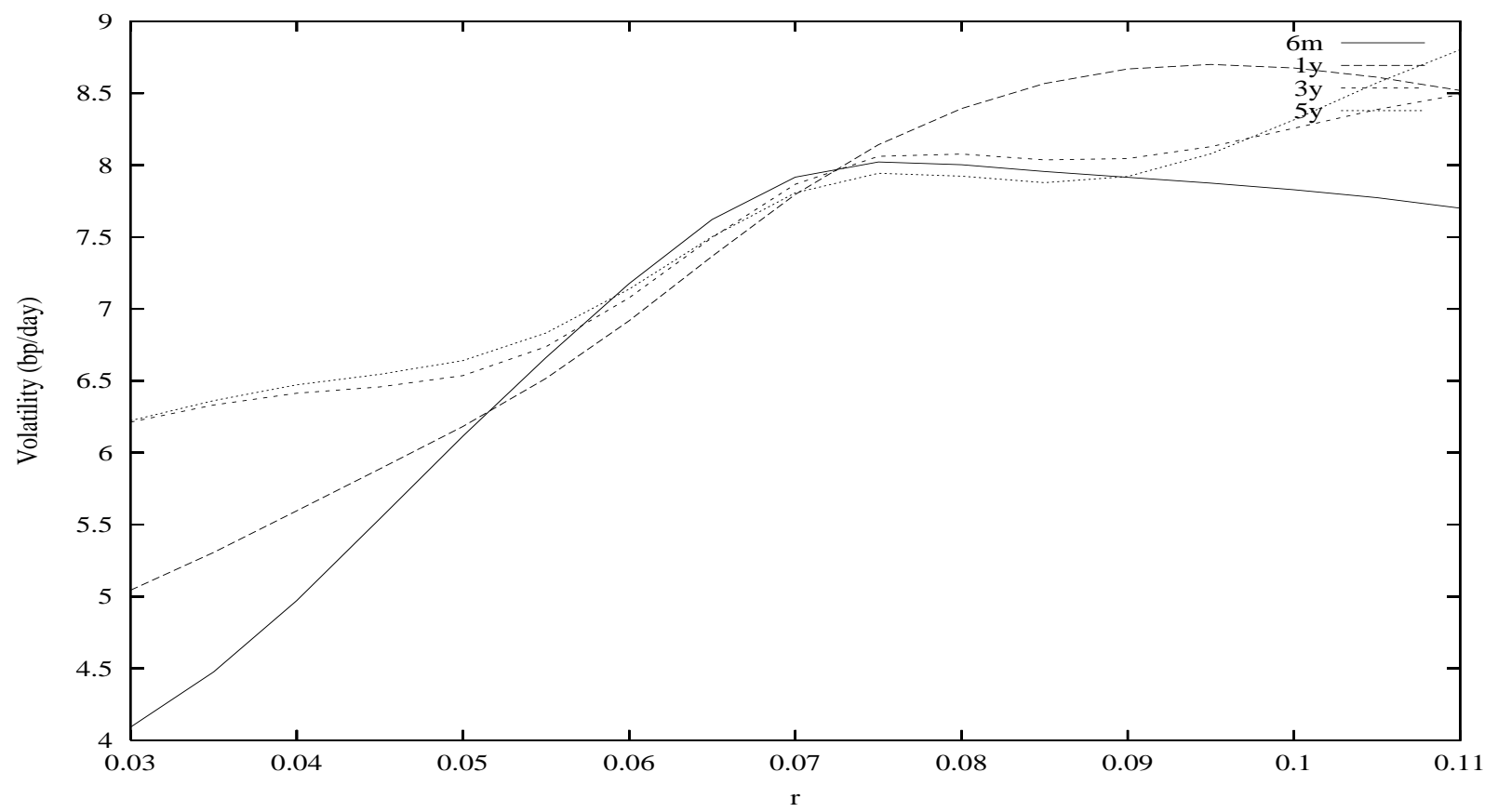

Figure 7: The volatility of the daily change in yields vs. the short rate, with the spread fixed at $2.75 \%$.

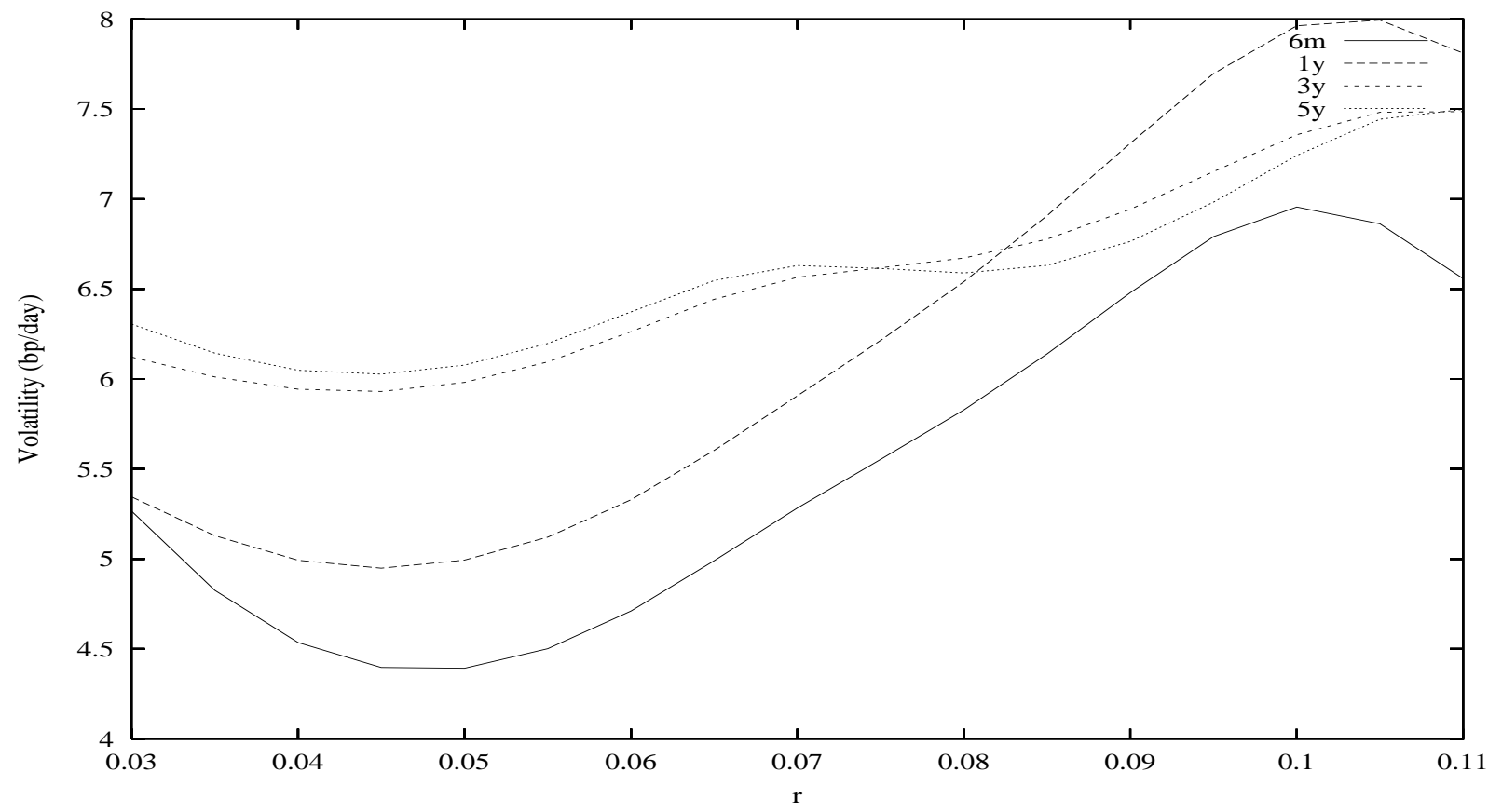

Figure 8: The volatility of the daily change in yields vs. the short rate, with the spread fixed at $1 \%$. 


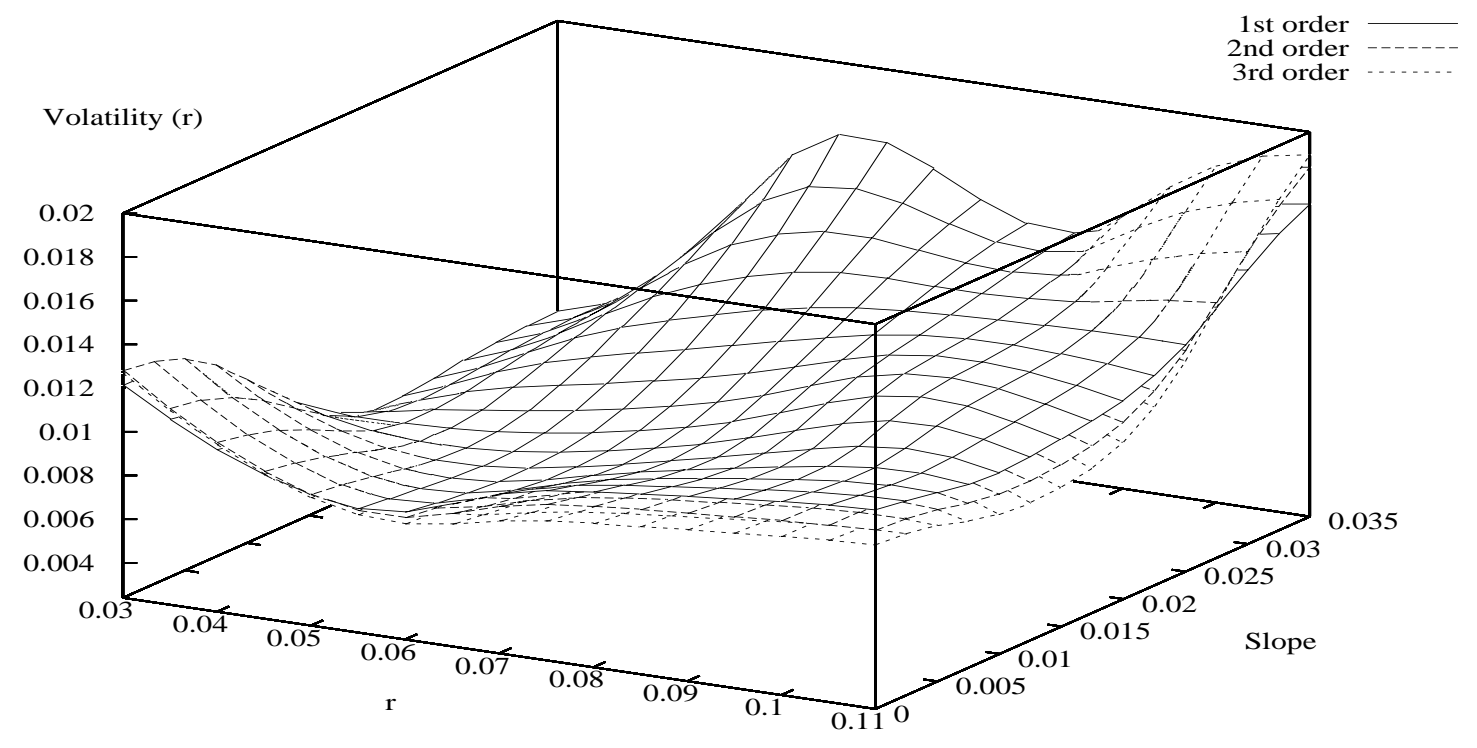

Figure 9: First, second, and third order approximations to the diffusion (annualized) of the short rate vs. the short rate and the slope of the term structure. 


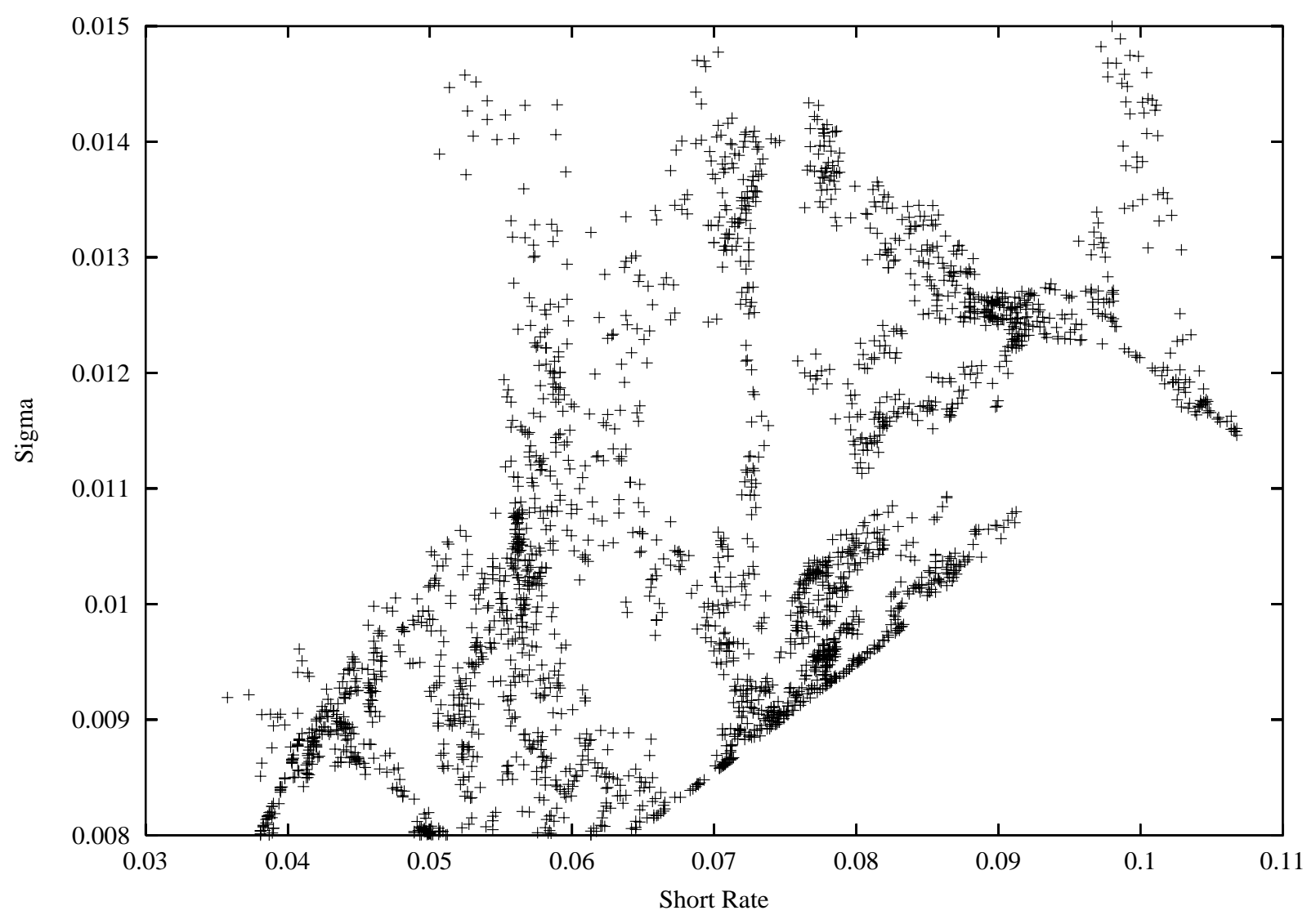

Figure 10: Scatter plot of the 3-month rate vs. the term structure volatility over the 19831996 period. 


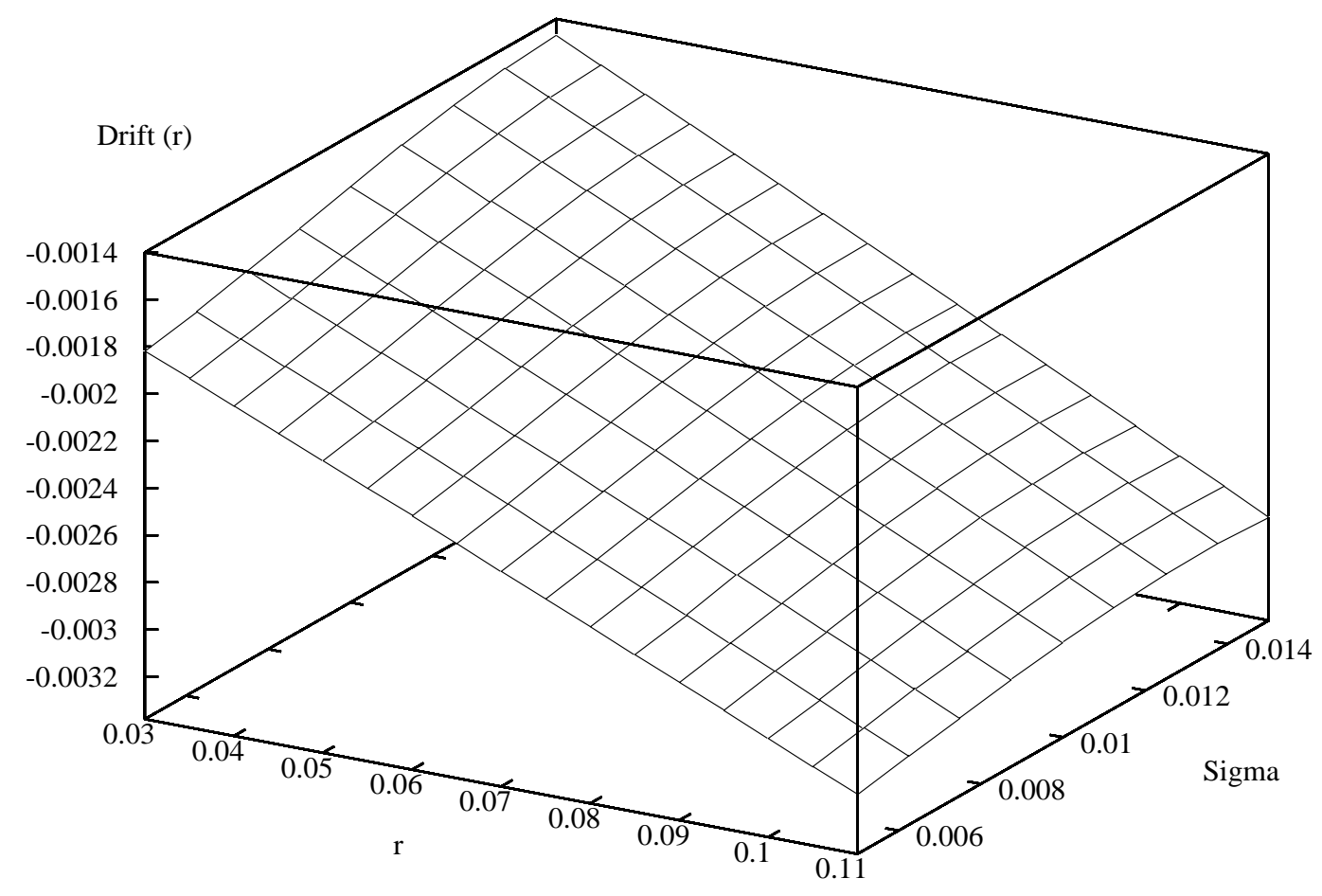

Figure 11: First order approximation to the drift (annualized) of the short rate vs. the short rate and the volatility of the term structure. 


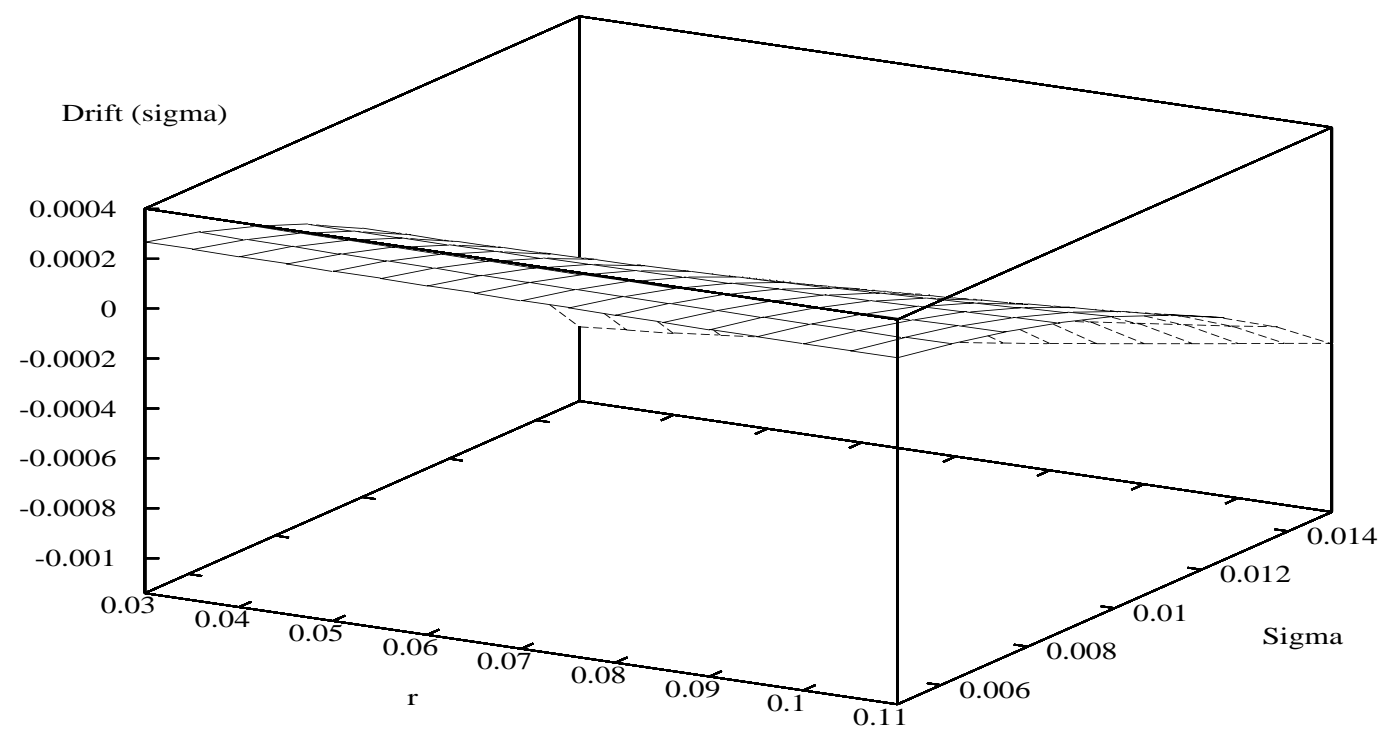

Figure 12: First order approximation to the drift (annualized) of the volatility vs. the short rate and the volatility of the term structure.

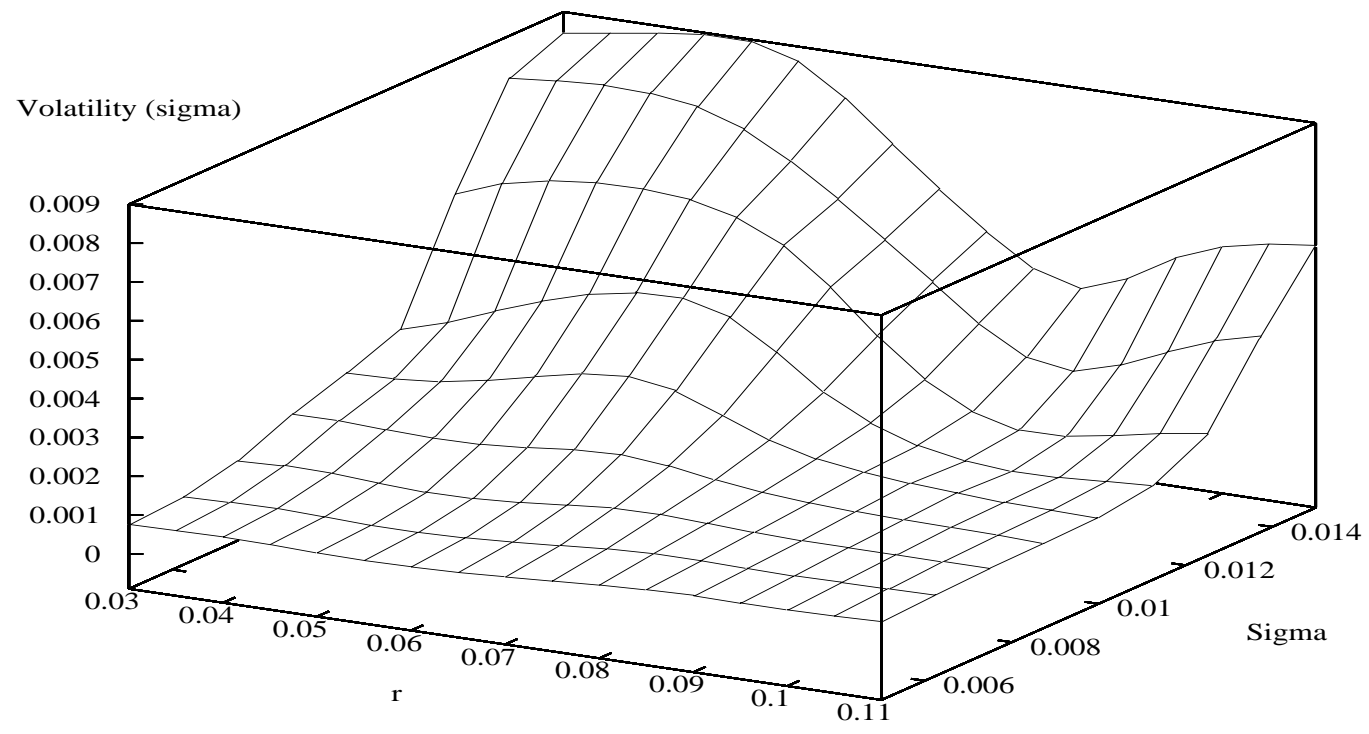

Figure 13: First order approximations to the diffusion (annualized) of the volatility vs. the short rate and the volatility of the term structure. 


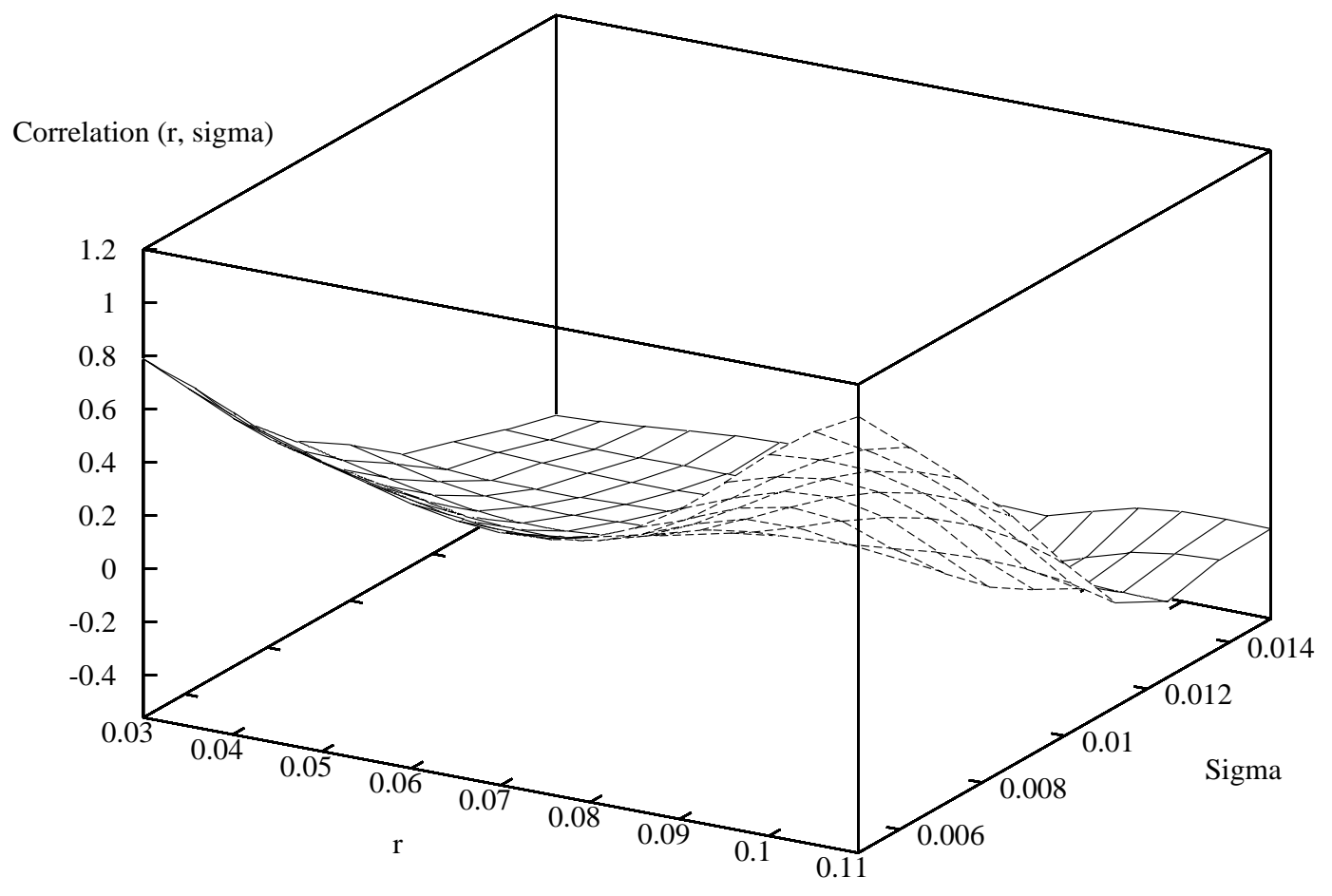

Figure 14: First order approximation to the correlation coefficient between changes in the short rate and the volatility vs. the short rate and the volatility of term structure. 


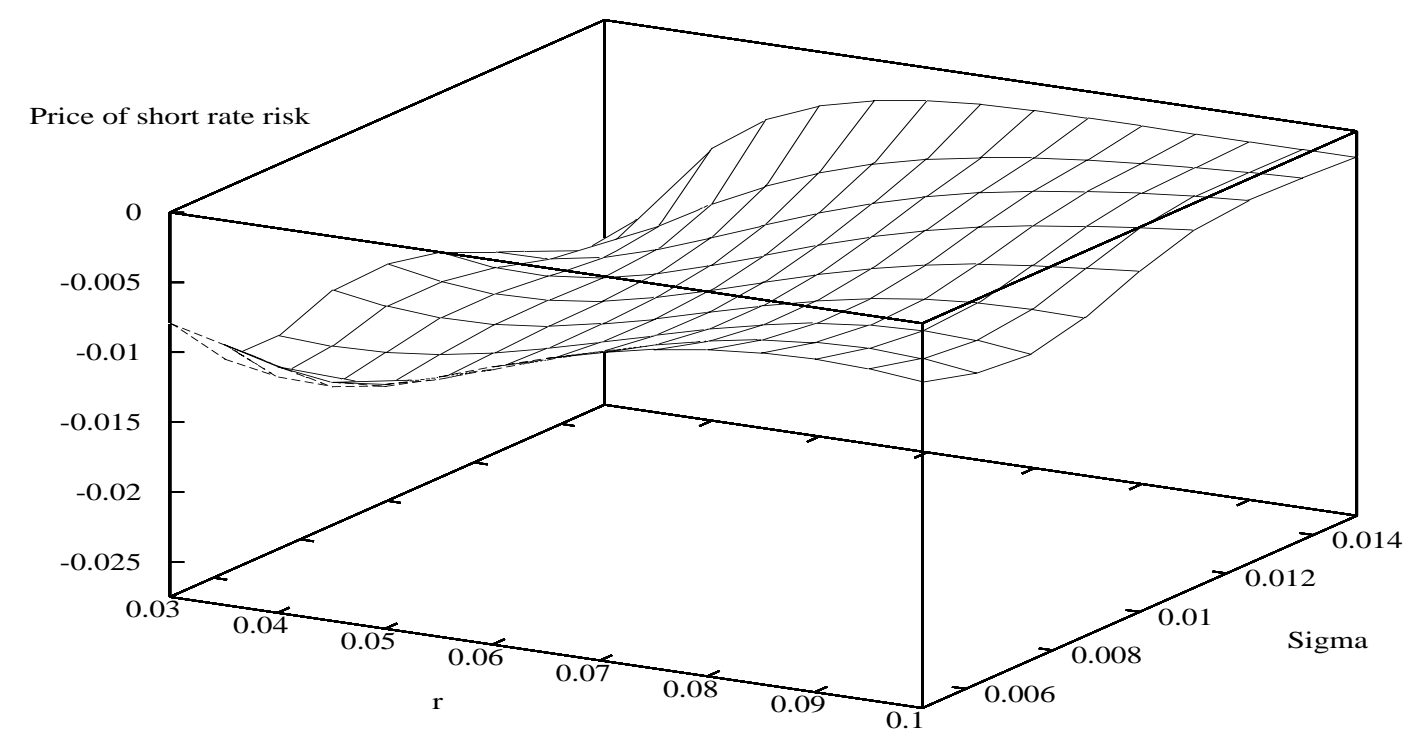

Figure 15: The price of short rate risk vs. the short rate and the volatility of the term structure.

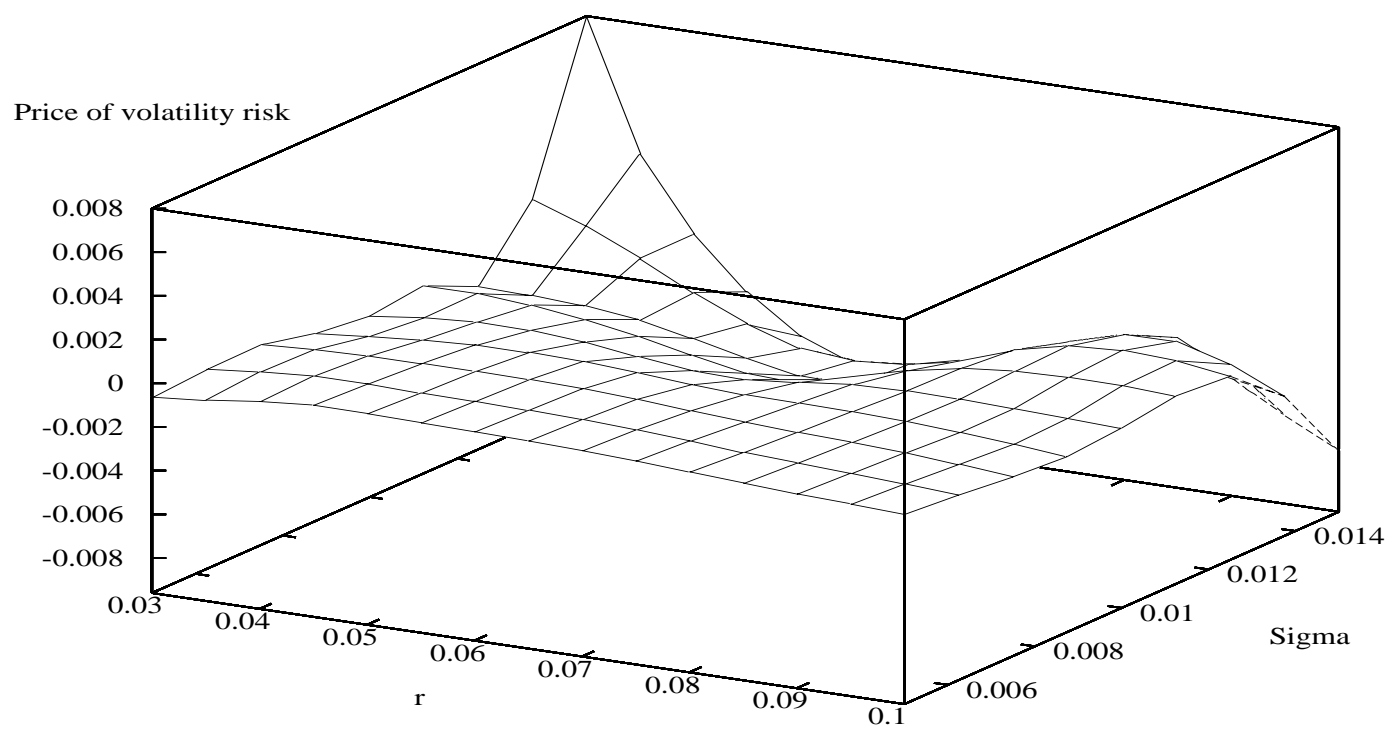

Figure 16: The price of volatility risk vs. the short rate and the volatility of the term structure. 


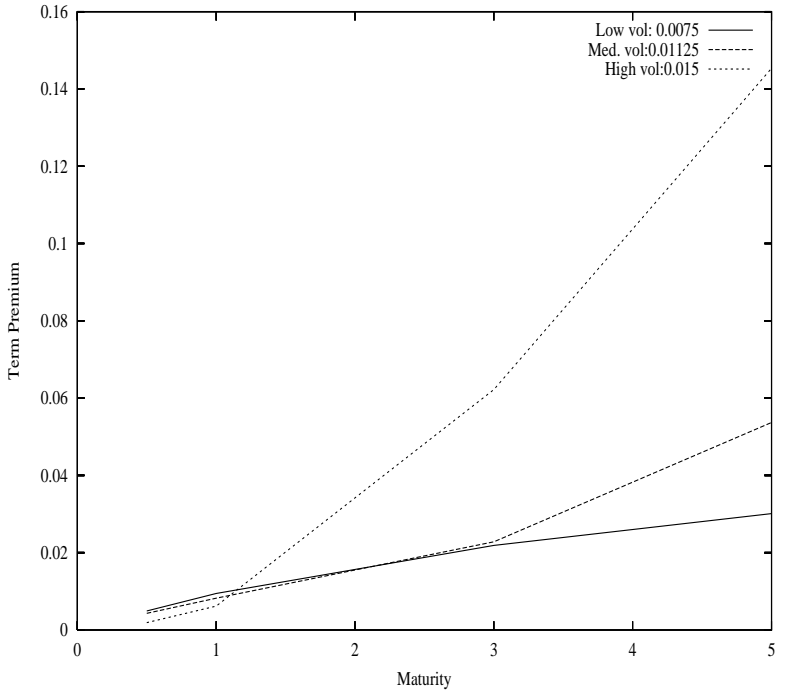

(a) $r=5.5 \%$

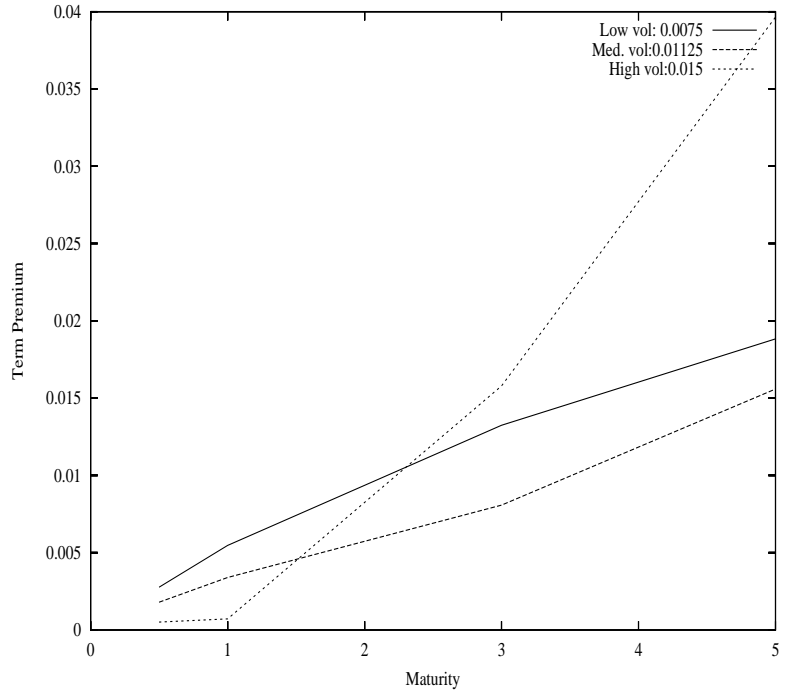

(b) $r=8.0 \%$

Figure 17: Term structure of term premia 